\title{
PLÜCKER VARIETIES AND HIGHER SECANTS OF SATO'S GRASSMANNIAN
}

\author{
JAN DRAISMA AND ROB H. EGGERMONT
}

\begin{abstract}
Every Grassmannian, in its Plücker embedding, is defined by quadratic polynomials. We prove a vast, qualitative, generalisation of this fact to what we call Plücker varieties. A Plücker variety is in fact a family of varieties in exterior powers of vector spaces that, like the Grassmannian, is functorial in the vector space and behaves well under duals. A special case of our result says that for each fixed natural number $k$, the $k$-th secant variety of any Plücker-embedded Grassmannian is defined in bounded degree independent of the Grassmannian. Our approach is to take the limit of a Plücker variety in the dual of a highly symmetric space known as the infinite wedge, and to prove that up to symmetry the limit is defined by finitely many polynomial equations. For this we prove the auxilliary result that for every natural number $p$ the space of $p$-tuples of infinite-by-infinite matrices is Noetherian modulo row and column operations. Our results have algorithmic counterparts: every bounded Plücker variety has a polynomial-time membership test, and the same holds for Zariski-closed, basis-independent properties of $p$-tuples of matrices.
\end{abstract}

\section{IntRodUCtion AND MAIN RESUlts}

To motivate our results, we first recall Grassmannians in their Plücker embeddings. For a natural number $p$ and a vector space $V$ over a field $K$, the Grassmannian of $p$-dimensional subspaces of $V$ lives in the projective space associated to the $p$-th exterior power $\bigwedge^{p} V$ of $V$. Let $\operatorname{Gr}(p, V) \subseteq \bigwedge^{p} V$ denote the affine cone over that Grassmannian. It consists of all pure tensors, i.e., those of the form $v_{1} \wedge \cdots \wedge v_{p}$ with each $v_{i} \in V$.

As $p$ and $V$ vary, the varieties $\operatorname{Gr}(p, V)$ satisfy two fundamental axioms. First, if $f: V \rightarrow W$ is a linear map, then the induced linear map $\bigwedge^{p} f: \bigwedge^{p} V \rightarrow \bigwedge^{p} W$ maps $\operatorname{Gr}(p, V)$ into $\operatorname{Gr}(p, W)$. In particular, $\operatorname{Gr}(p, V)$ is stable under linear automorphisms of $V$. Second, if $V$ has dimension $p+n$ with $p, n \geq 0$, then the natural linear isomorphism $\bigwedge^{p} V \rightarrow \bigwedge^{n} V^{*}$ (natural, that is, up to a scalar) maps $\operatorname{Gr}(p, V)$ into $\operatorname{Gr}\left(n, V^{*}\right)$. Indeed, its projectivisation maps a point in the first Grassmannian, representing a $p$-dimensional subspace $U$ of $V$, to the point in the second Grassmannian that represents the annihilator $U^{0}$ of $U$ in $V^{*}$.

In this paper, we consider a general family $\left\{\mathbf{X}_{p}(V) \subseteq \bigwedge^{p} V\right\}_{p, V}$ of closed subvarieties of exterior powers satisfying the same two axioms. We call such a family a Plücker variety; see Section 2 for a formal definition. Thus a Plücker variety is not a single variety but rather a rule $\mathbf{X}$ that assigns to a number $p$ and a finitedimensional $K$-vector space $V$ a closed subvariety $\mathbf{X}_{p}(V) \subseteq \bigwedge^{p} V$, subject to the axioms above. A Plücker variety is called bounded if $\mathbf{X}_{2}(V) \subsetneq \Lambda^{2} V$ for at least

Both authors are supported by the first author's Vidi grant from the Netherlands Organisation for Scientific Research (NWO). 
some finite-dimensional vector space $V$ (and hence, as we will see, for all $V$ of sufficiently high dimension).

To avoid the anomaly that the Zariski topology becomes discrete, we will assume throughout that the ground field $K$ of our vector spaces and varieties is infinite. Our main theorem is then as follows.

Theorem 1.1 (Main Theorem). For any bounded Plücker variety $\mathbf{X}$ there exists a $p_{0} \in \mathbb{Z}_{\geq 0}$ and a finite-dimensional vector space $V_{0}$ such that all instances $\mathbf{X}_{p}(V)$ of $\mathbf{X}$ are defined set-theoretically by polynomial equations obtained from those of $\mathbf{X}_{p_{0}}\left(V_{0}\right)$ by pulling back along sequences of linear maps of the two types above. In particular, $\mathbf{X}_{p}(V)$ is defined set-theoretically by equations of bounded degree.

As we will see below, the class of bounded Plücker varieties is closed under taking secant varieties. So a direct consequence of our main theorem is the following.

Corollary 1.2. For any $k$, there exists a $d$ such that for any natural number $p$ and any finite-dimensional $K$-vector space $V$, the $k$-th secant variety of $\operatorname{Gr}(p, V)$ inside $\wedge^{p} V$ is defined set-theoretically by polynomials of degree at most $d$.

For instance, consider the Grassmannian itself. It is well known that $\operatorname{Gr}(p, V)$ is defined by certain equations of degree two called Plücker relations. But in fact, up to coordinate changes a single Plücker relation suffices. Indeed, a set of defining equations for $\operatorname{Gr}(p, V)$ can be found simply by taking pullbacks of the Klein quadric defining $\operatorname{Gr}\left(2, K^{4}\right)$ KPRS08. In general, this set does not generate the full ideal of the Grassmannian. But it does show that one can test membership of $\operatorname{Gr}(p, V)$ using a single type of equation; our result above generalises this statement to Plücker varieties. It has the following algorithmic consequence.

Theorem 1.3. For any bounded Plücker variety $\mathbf{X}$ there exists a polynomial-time algorithm that on input $d, p \in \mathbb{Z}_{\geq 0}$ and $\omega \in \bigwedge^{p} K^{d}$ tests whether $\omega \in \mathbf{X}_{p}\left(K^{d}\right)$.

Here $\omega$ is given in a non-sparse encoding, and polynomial-time refers to the number of arithmetic operations over the field generated by the entries of $\omega$ (this field, unlike $K$, may be finite). Moreover, if that field is the rational numbers or a more general number field, then even the bit-complexity of the algorithm is polynomial.

Remark 1.4. We stress that in Theorem 1.3 the Plücker variety $X$ is fixed (for instance, equal to the third secant variety of the Grassmannian) and that the proof of the theorem is non-constructive in the sense that the $p_{0}$ and $V_{0}$ from Theorem 1.1 have to be built into the algorithm. We do not claim that $p_{0}$ and $V_{0}$ themselves can be found efficiently.

This paper is motivated by two main goals. The first is to understand varieties built up from Grassmannians by operations such as joins, secant varieties, and tangential varieties. All of these are examples of bounded Plücker varieties. For instance, if $\mathbf{X}, \mathbf{Y}$ are bounded Plücker varieties, then the rule $\mathbf{X}+\mathbf{Y}$ that assigns to $p, V$ the Zariski closure of $\left\{x+y \mid x \in \mathbf{X}_{p}(V), y \in \mathbf{Y}_{p}(V)\right\} \subseteq \bigwedge^{p} V$ is again a bounded Plücker variety, called the join of $\mathbf{X}$ and $\mathbf{Y}$, to which our theorem applies. In the special case where $\mathbf{Y}=\mathbf{X}$, the join is called the secant variety of $\mathbf{X}$, and higher secant varieties are obtained by repeatedly taking the join with $\mathbf{X}$. Quite a bit is known about the dimensions of these secant varieties [CGG05, BDdG07, but almost nothing is known about their defining equations. 
The second goal is to develop an exterior-power analogue of Snowden's theory of $\Delta$-varieties Sno13. That theory concerns varieties (or schemes) of ordinary tensors, rather than alternating tensors. For ordinary tensors, the analogues of our results are established in DK14. Also, for ordinary tensors, many more concrete results are known on equations for the first few higher secant varieties Str83, LM04, Rai12, Qi13. For symmetric tensors, also quite a lot is known about equations for secant varieties (see, e.g., BB14, LO13), but we do not yet know whether a symmetric counterpart to our Theorems 1.1 and 1.3 exists.

The proofs of both theorems are non-constructive. In particular, we do not find new equations for secant or tangential varieties of Grassmannians other than pullbacks of Pfaffians. Finding explicit equations is an art that involves sophisticated techniques from representation theory [LO13, MM14]. Instead, we will establish the fundamental fact that up to symmetry, finitely many equations suffice.

The key notion in our approach is Noetherianity up to symmetry. A topological space on which a group $G$ acts by means of homeomorphisms is said to be $G$-Noetherian (or equivariantly Noetherian if $G$ is clear from the context) if every descending chain of closed, $G$-stable subsets stabilises. We refer to $\mathrm{CDD}^{+} 14$, Chapter 2] for a gentle introduction. There is currently a surge of activity on related stabilisation issues in algebraic geometry and its applications, e.g. in algebraic statistics HS12, Dra10, DE15, invariant theory [HMSV09, representation theory CEF15, and commutative algebra SS12a, SS12b, DEKL13. The following new example of this phenomenon will play a fundamental role in the proofs of our theorems, but is likely to be useful in other applications. Let Mat $\mathrm{T}_{\mathbb{N}, \mathbb{N}}$ denote the (uncountably dimensional) space of all $\mathbb{N} \times \mathbb{N}$-matrices over $K$. Similarly, for $n, m \in \mathbb{Z}_{\geq 0}$ define $\operatorname{Mat}_{\mathbb{N}, n}$ and $\mathrm{Mat}_{m, \mathbb{N}}$. Consider the group $\mathrm{GL}_{\mathbb{N}}:=\bigcup_{n \in \mathbb{N}} \mathrm{GL}_{n}$ of all invertible matrices having zeroes almost everywhere outside the diagonal (i.e. everywhere outside the diagonal except in a finite number of positions) and ones almost everywhere on the diagonal. One copy of this group acts by left multiplication on $\mathrm{Mat}_{\mathbb{N}, \mathbb{N}}$ and $\mathrm{Mat}_{\mathbb{N}, n}$ and trivially on $\mathrm{Mat}_{m, \mathbb{N}}$, and one copy acts by right multiplication on $\operatorname{Mat}_{\mathbb{N}, \mathbb{N}}$ and $\operatorname{Mat}_{m, \mathbb{N}}$ and trivially on Mat $\mathbb{N}, n$. For any $p, d \in \mathbb{Z}_{\geq 0}$, consider the Cartesian product

$$
A_{p, n, m, d}:=\left(\operatorname{Mat}_{\mathbb{N}, \mathbb{N}}\right)^{p} \times \operatorname{Mat}_{\mathbb{N}, n} \times \operatorname{Mat}_{m, \mathbb{N}} \times K^{d},
$$

equipped with the Zariski topology in which closed sets are given by polynomials in the entries of the $p+2$ matrices and the coordinates on the latter $K^{d}$. Let $\mathrm{GL}_{\mathbb{N}} \times \mathrm{GL}_{\mathbb{N}}$ act diagonally, and trivially on $K^{d}$.

Theorem 1.5. For any $p, n, m, d \in \mathbb{Z}_{>0}$, the topological space $A_{p, n, m, d}$ is equivariantly Noetherian with respect to $\mathrm{GL}_{\mathbb{N}} \times \mathrm{GL}_{\mathbb{N}}$. In other words, every $\mathrm{GL}_{\mathbb{N}} \times \mathrm{GL}_{\mathbb{N}}-$ stable closed subset can be characterised as the common zero set of finitely many $\mathrm{GL}_{\mathbb{N}} \times \mathrm{GL}_{\mathbb{N}}$-orbits of polynomial equations.

We do not know whether the corresponding ideal-theoretic statement also holds, i.e., whether each $\mathrm{GL}_{\mathbb{N}} \times \mathrm{GL}_{\mathbb{N}}$-stable ideal in the coordinate ring of the variety in the theorem is generated by finitely many orbits of polynomials. We return to this question in Section 9

The remainder of this paper is organised as follows. In Section 2 , we give a formal definition of Plücker varieties and discuss the boundedness condition. In Section 3 we introduce the infinite wedge (or rather, its charge-zero part), and in Section 4 we construct, for any Plücker variety, a limit object in the space dual to 
the infinite wedge. In the case of the Grassmannian, this limit is known as (the charge-zero part of) Sato's Grassmannian SS83, SW85, VMP98. For a general bounded Plücker variety, the limit lies in the variety defined by certain Pfaffians, which we describe in Section 5. Then, in Section 6 we show that these Pfaffian varieties are equivariantly Noetherian with respect to a group that preserves the limit of any Plücker variety. In particular, this shows that the limit is defined by finitely many equations under that group. Finally, in Section 7, we go back to finitedimensional instances of a Plücker variety and complete the proof of Theorems 1.1 and 1.3 The Noetherianity in Section 6 is proved using the auxilliary Theorem 1.5 . whose proof we defer to Section 8. Finally, in Section 9, we discuss a number of open questions.

\section{PlüCKER VARIETIES AND BOUNDEDNESS}

Throughout this paper, we work over an infinite field $K$, and we use the convention $\mathbb{N}=\{1,2,3, \ldots\}$ and $[n]:=\{1, \ldots, n\}$ for $n \in \mathbb{Z}_{\geq 0}$. By a (finite-dimensional) variety we mean a Zariski-closed subset of a finite-dimensional vector space over $K$. Sometimes we will stress the closedness and say closed subvariety.

If $V$ is a vector space with basis $v_{1}, \ldots, v_{m}$, then $\Lambda^{p} V$ has a basis consisting of the vectors $v_{I}:=v_{i_{1}} \wedge \cdots \wedge v_{i_{p}}$ where $I=\left\{i_{1}<\ldots<i_{p}\right\}$ runs over all $p$-subsets of $[m]$. We will call this the standard basis of $\Lambda^{p} V$ relative to the given basis $\left\{v_{i}\right\}_{i}$. We always identify $\left(\bigwedge^{p} V\right)^{*}$ with $\bigwedge^{p}\left(V^{*}\right)$ via the map from the latter space to the former that sends $x_{1} \wedge \cdots \wedge x_{p}$ to the linear function determined by

$$
v_{1} \wedge \cdots \wedge v_{p} \mapsto \sum_{\pi \in S_{p}} \operatorname{sgn}(\pi) \prod_{i=1}^{p} x_{i}\left(v_{\pi(i)}\right) .
$$

Given a vector space $V$ of dimension $p+n$ with $p, n \in \mathbb{Z}_{\geq 0}$, and choosing an isomorphism $\psi: \bigwedge^{p+n} V \rightarrow K$, we obtain an isomorphism $\star: \bigwedge^{p} V \rightarrow \bigwedge^{n} V^{*}$ defined by $\star(\omega)\left(\omega^{\prime}\right)=\psi\left(\omega \wedge \omega^{\prime}\right)$. The map $\star$ is well-defined up to choice of $\psi$. We call such a map a Hodge dual. Classically, Hodge duals go one step further in identifying $\bigwedge^{n} V^{*}$ with $\bigwedge^{n} V$ by means of a symmetric bilinear form on $V$, but we will not do this. Note that if $\star: \bigwedge^{p} V \rightarrow \bigwedge^{n} V^{*}$ is a Hodge dual, then so is its inverse $\star^{-1}: \bigwedge^{n} V^{*} \rightarrow \bigwedge^{p} V$ and its dual $\star^{*}: \bigwedge^{n} V \rightarrow \bigwedge^{p} V^{*}$.

Definition 2.1. A Plücker variety is a sequence $\mathbf{X}=\left(\mathbf{X}_{p}\right)_{p \in \mathbb{Z} \geq 0}$ of functors from the category of finite-dimensional vector spaces to the category of varieties satisfying the following axioms:

(1) For all vector spaces $V$ and for all $p \in \mathbb{Z}_{\geq 0}$, the variety $\mathbf{X}_{p}(V)$ is a closed subvariety of $\bigwedge^{p} V$.

(2) For all $p \in \mathbb{Z}_{\geq 0}$ and for all linear maps $\varphi: V \rightarrow W$, the map $\mathbf{X}_{p}(\varphi)$ : $\mathbf{X}_{p}(V) \rightarrow \mathbf{X}_{p}(W)$ is the restriction of $\bigwedge^{p} \varphi$ to $\mathbf{X}_{p}(V)$.

(3) If $V$ is a vector space of dimension $n+p$ with $n, p \in \mathbb{Z}_{\geq 0}$, and $\star: \bigwedge^{p} V \rightarrow$ $\bigwedge^{n} V^{*}$ is a Hodge dual, then $\star \operatorname{maps} \mathbf{X}_{p}(V)$ into $\mathbf{X}_{n}\left(V^{*}\right)$.

Given a Plücker variety $\mathbf{X}$, a variety of the form $\mathbf{X}_{p}(V)$ for a specific choice of $p$ and $V$ is called an instance of the Plücker variety $\mathbf{X}$.

Example 2.2. The following constructions give a rich source of Plücker varieties.

(1) $\mathbf{X}_{p}(V):=\bigwedge^{p} V, \mathbf{X}_{p}(V):=\emptyset, \mathbf{X}_{p}(V):=\{0\}$ are Plücker varieties. 
(2) $\mathbf{X}_{p}(V):=\operatorname{Gr}(p, V)$ is the (cone over the) Grassmannian; we will see that this is the smallest non-zero Plücker variety.

(3) Given Plücker varieties $\mathbf{X}$ and $\mathbf{Y}$, the rules $\mathbf{X} \cap \mathbf{Y}$ and $\mathbf{X} \cup \mathbf{Y}$ defined in the obvious manner are Plücker varieties; and

(4) Similarly, the join $\mathbf{X}+\mathbf{Y}$ and the tangential variety $\tau \mathbf{X}$ defined by

$$
\begin{aligned}
& (\mathbf{X}+\mathbf{Y})_{p}(V):=\overline{\left\{x+y \mid x \in \mathbf{X}_{p}(V), y \in \mathbf{Y}_{p}(V)\right\}} \text { and } \\
& (\tau \mathbf{X})_{p}(V):=\overline{\left\{x \mid x \in \ell \text { for some line } \ell \text { tangent to } \mathbf{X}_{p}(V) \text { at a smooth point }\right\}}
\end{aligned}
$$

are Plücker varieties.

The second axiom implies that for any Plücker variety, any $p \in \mathbb{Z}_{\geq 0}$ and any $V$, the instance $\mathbf{X}_{p}(V)$ is stable under the action of $\mathrm{GL}(V)$. Moreover, it is stable under multiplication with scalars: if $\star: \bigwedge^{p} V \rightarrow \bigwedge^{n} V^{*}$ is a Hodge dual, then for any scalar $t \in K$, we have $t \mathbf{X}_{p}(V)=\left(t \star^{-1}\right) \star \mathbf{X}_{p}(V) \subseteq \mathbf{X}_{p}(V)$, because $t \star^{-1}$ is a Hodge dual, as well. Hence, provided that it is non-empty, $\mathbf{X}_{p}(X)$ is the affine cone over a projective variety. To avoid having to deal with rational maps, we work with the cone rather than the projective variety.

When combined, the axioms for Plücker varieties give further maps connecting instances of $\mathbf{X}$. The following lemmas extract two fundamental types of such maps.

Lemma 2.3 (Tensoring). Let $W$ be a finite-dimensional vector space, let $V$ be a codimension-one subspace of $W$, and write $W=V \oplus\langle w\rangle$. Then for any Plücker variety $\mathbf{X}$ and any $p \in \mathbb{Z}_{\geq 0}$ with $p \leq \operatorname{dim} V$ the map

$$
\bigwedge^{p} V \rightarrow \bigwedge^{p+1} W, \quad \omega \mapsto \omega \wedge w
$$

maps $\mathbf{X}_{p}(V)$ into $\mathbf{X}_{p+1}(W)$.

Proof. Set $n:=\operatorname{dim} V-p$. The map in the lemma is the composition

$$
\bigwedge^{p} V \stackrel{\star_{1}}{\longrightarrow} \bigwedge^{n} V^{*} \stackrel{\Lambda^{n} \varphi}{\longrightarrow} \bigwedge^{n} W^{*} \stackrel{\star_{2}}{\longrightarrow} \bigwedge^{p+1} W
$$

where $\star_{1}, \star_{2}$ are suitable Hodge duals and $\varphi: V^{*} \rightarrow W^{*}$ is the map that extends a linear function by zero on $\langle w\rangle$.

Lemma 2.4 (Contraction). In the setting of the previous lemma, let $\iota$ denote the embedding $V \rightarrow W$, so that $\iota^{*}: W^{*} \rightarrow V^{*}$ is restriction of linear functions. Then the linear map determined by

$$
\begin{aligned}
\bigwedge^{p+1} W^{*} & \rightarrow \bigwedge^{p} V^{*} \\
x_{1} \wedge \cdots \wedge x_{p+1} & \mapsto \sum_{i=1}^{p+1}(-1)^{p+1-i} x_{i}(w) \cdot\left(\bigwedge^{p} \iota^{*}\right)\left(x_{1} \wedge \cdots \widehat{x_{i}} \cdots \wedge x_{p+1}\right)
\end{aligned}
$$

maps $\mathbf{X}_{p+1}\left(W^{*}\right)$ into $\mathbf{X}_{p}\left(V^{*}\right)$.

Proof. First, we claim that this map is the dual of the map in the previous lemma. Indeed, evaluating $x_{1} \wedge \cdots \wedge x_{p+1}$ on $v_{1} \wedge \cdots \wedge v_{p} \wedge w$ yields the same as evaluating the right-hand side above on $v_{1} \wedge \cdots \wedge v_{p}$. Now the desired result follows by taking the dual maps in Diagram (1).

Here is a first illustration of how a single instance of a Plücker variety may determine all of it. 
Lemma 2.5. Let $\mathbf{X}$ be a Plücker variety. Then $\mathbf{X}_{0}(K)=\{0\}$ if and only if $\mathbf{X}_{p}(V)=\{0\}$ for all $p$ and $V$.

Proof. The implication $\Leftarrow$ is immediate. For the implication $\Rightarrow$, pick a non-zero vector $\omega \in \mathbf{X}_{p}(V)$, let $v_{1}, \ldots, v_{p}$ be a basis of $V$, and assume that the coefficient in $\omega$ of the standard basis vector $v_{i_{1}} \wedge \cdots \wedge v_{i_{p}}$ is non-zero. Set $W:=\left\langle v_{i_{1}}, \ldots, v_{i_{p}}\right\rangle$ and let $\pi: V \rightarrow W$ be the projection along the remaining basis vectors. Then $\omega^{\prime}:=\left(\bigwedge^{p} \pi\right) \omega$ is a non-zero element of $\mathbf{X}_{p}(W)$. Next, apply $\star: \bigwedge^{p} W \rightarrow \bigwedge^{0} W^{*}$ to $\omega^{\prime}$ to obtain a non-zero $\omega^{\prime \prime} \in \mathbf{X}_{0}\left(W^{*}\right)$. Finally, the zeroth exterior power of any linear map $W^{*} \rightarrow K$ is an isomorphism and maps $\omega^{\prime \prime}$ to a non-zero element of $\mathbf{X}_{0}(K)$.

It follows that if $\mathbf{X}_{0}(V)=\{0\}$ for some $V$, then the full Plücker variety is zero. Moreover, since the only closed subvarieties of $V$ that are $\mathrm{GL}(V)$-stable are $V$ and $\{0\}$, we find that if $\mathbf{X}_{1}(V) \neq V$ for some $V$, then $\mathbf{X}_{1}(V)=\{0\}$ and consequently, the full Plücker variety is zero. So only Plücker varieties with $\mathbf{X}_{1}(V)=V$ for all $V$ are of interest to us.

Next, GL $(V)$ has exactly $\left\lfloor\frac{\operatorname{dim} V}{2}\right\rfloor$ orbits on $\bigwedge^{2} V$. Indeed, any $\omega$ in this space is of the form $v_{1} \wedge v_{2}+\ldots+v_{2 r-1} \wedge v_{2 r}$ with $v_{1}, \ldots, v_{2 r}$ linearly independent, so that $2 r \leq \operatorname{dim} V$. The number $r$ is called the rank of $\omega$, denoted $\mathrm{rk} \omega$. It is half the rank of the skew-symmetric matrix $\left(\left(x_{i} \wedge x_{j}\right)(\omega)\right)_{i j}$ where $x_{i}, x_{j}$ range over a basis of $V^{*}$. For $r$ in this range, define

$$
Y^{r}(V):=\{\omega \mid \operatorname{rk}(\omega) \leq r\} .
$$

As we assume that $K$ is infinite, the $Y^{r}(V)$ are the only Zariski-closed, $\operatorname{GL}(V)$ stable subsets of $\bigwedge^{2} V$.

Lemma 2.6. Let $\mathbf{X}$ be a Plücker variety. Suppose that there exists a vector space $V$ such that $\mathbf{X}_{2}(V)=Y^{r}(V) \neq \bigwedge^{2} V$. Then for all vector spaces $W$, we have $\mathbf{X}_{2}(W)=Y^{r}(W)$.

Proof. Suppose that $\mathbf{X}_{2}(W)$ contains $\omega$ s with rank strictly exceeding $r$. Since it is closed and $\mathrm{GL}(W)$-stable, it contains an $\omega$ of rank equal to $r+1$. Write $\omega=w_{1} \wedge w_{2}+\ldots+w_{2 r+1} \wedge w_{2 r+2}$. Note that $V$ has dimension at least $2 r+2$, because $Y^{r}(V) \neq \bigwedge^{2} V$. Let $\varphi: W \rightarrow V$ be a linear map that maps $w_{1}, \ldots, w_{2 r+2}$ to linearly independent elements. Then $\bigwedge^{2} \varphi(\omega)$ has rank $r+1$. This gives a contradiction, since $\bigwedge^{2} \varphi(\omega) \in \mathbf{X}_{2}(V)$. We conclude $\mathbf{X}_{2}(W) \subseteq Y^{r}(W)$.

Conversely, let $\omega \in Y^{r}(W)$ and write $\omega=w_{1} \wedge w_{2}+\ldots+w_{2 r^{\prime}-1} \wedge w_{2 r^{\prime}}$ for some $r^{\prime} \leq r$, with $w_{1}, \ldots, w_{2 r^{\prime}}$ linearly independent. Let $v_{1}, \ldots, v_{2 r^{\prime}} \in V$ be linearly independent, and let $\varphi: V \rightarrow W$ be a linear map that maps $v_{i}$ to $w_{i}$. We have $v_{1} \wedge v_{2}+\ldots+v_{2 r^{\prime}-1} \wedge v_{2 r^{\prime}} \in \mathbf{X}_{2}(V)$, and its image under $\wedge^{2} \varphi$ is $\omega$, hence $\omega \in \mathbf{X}_{2}(W)$. We conclude $\mathbf{X}_{2}(W)=Y^{r}(W)$.

Dually, define $Y^{r, \star}(V):=\star Y^{r}\left(V^{*}\right) \subseteq \bigwedge^{\operatorname{dim} V-2} V$. Note that $Y^{r, \star}(V)$ is independent of choice of Hodge dual. By taking Hodge duals, we get matching statements in $\bigwedge^{\operatorname{dim} V-2} V$ for each $V$.

Lemma 2.7. The only closed $\mathrm{GL}(V)$-stable subvarieties of $\bigwedge^{\operatorname{dim} V-2} V$ are the varieties $Y^{r, \star}(V)$. Moreover, if $\mathbf{X}$ is a Plücker variety, and if $\mathbf{X}_{\operatorname{dim} V-2}(V)=Y^{r, \star}(V) \neq$ $\bigwedge^{\operatorname{dim} V-2} V$ for some $V$, then $\mathbf{X}_{\operatorname{dim} W-2}(W)=Y^{r, \star}(W)$ for all $W$. 
Definition 2.8. A Plücker variety $\mathbf{X}$ is called bounded if there exists some $V$ for which $\mathbf{X}_{2}(V) \neq \bigwedge^{2} V$. In this case, there exists a unique $r$ such that $\mathbf{X}_{2}(V)=Y^{r}(V)$ for all $V$, called the rank of the Plücker variety.

By the above, the rank also satisfies $\mathbf{X}_{\operatorname{dim} V-2}(V)=Y^{r, \star}(V)$ for all $V$ of dimension at least 2. Note that the Grassmannian is a bounded Plücker variety of rank 1, and that the constructions in Example 2.2 all preserve the class of bounded Plücker varieties. For example, the rank of the join $\mathbf{X}+\mathbf{Y}$ is at most (and in fact equal to) the sum of the ranks of $\mathbf{X}$ and $\mathbf{Y}$, and the rank of the tangential variety $\tau \mathbf{X}$, being contained in the secant variety $\mathbf{X}+\mathbf{X}$, is at most twice the rank of $\mathbf{X}$. This shows that all Plücker varieties of direct interest to us are bounded.

\section{The INFINITE WEDGE AND ITS DUAL}

In this section we introduce the infinite wedge. We start with a countabledimensional vector space

$$
V_{\infty}=\left\langle\ldots, x_{-3}, x_{-2}, x_{-1}, x_{1}, x_{2}, x_{3}, \ldots\right\rangle
$$

in which the $x_{i}, i \in-\mathbb{N} \cup \mathbb{N}$ are a basis. Note that we skip 0 , which makes the setup symmetric around zero. In the literature on the infinite wedge, this symmetry is achieved by labelling with half-integers, and in decreasing order [BO00, RZ13. Formulas from representation theory and integrable systems depend on this convention. But we will not need any of those formulas, so we take the liberty to simplify the notation and label with $-\mathbb{N} \cup \mathbb{N}$ instead.

For any $n \in \mathbb{Z}_{\geq 0}$ (for "negative") and $p \in \mathbb{Z}_{\geq 0}$ (for "positive") let $V_{n, p}$ be the $(n+p)$-dimensional subspace

$$
V_{n, p}:=\left\langle x_{-n}, \ldots, x_{-2}, x_{-1}, x_{1}, x_{2}, \ldots, x_{p}\right\rangle .
$$

We arrange the exterior powers $\bigwedge^{p} V_{n, p}$ into a two-dimensional commutative diagram as follows.

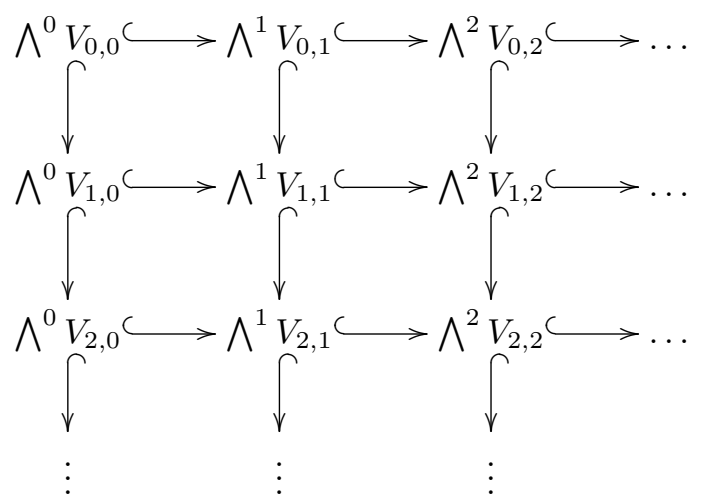

Here the vertical maps $\bigwedge^{p} V_{n, p} \rightarrow \bigwedge^{p} V_{n+1, p}$ are just the $p$-th exterior powers of the embeddings $V_{n, p} \rightarrow V_{n+1, p}$, and the horizontal maps $\bigwedge^{p} V_{n, p} \rightarrow \bigwedge^{p+1} V_{n, p+1}$ are given by $\omega \mapsto \omega \wedge x_{p+1}$. Note that both of these maps are injective. We will always identify $\bigwedge^{p} V_{n, p}$ with a subspace of $\bigwedge^{p^{\prime}} V_{n^{\prime}, p^{\prime}}$ for any $n^{\prime} \geq n$ and $p^{\prime} \geq p$ by means of the appropriate sequence of these maps.

If $x_{I}=x_{i_{1}} \wedge \cdots \wedge x_{i_{p}}$ is a standard basis element of $\wedge^{p} V_{n, p}$, then the subset $I=\left\{i_{1}<\ldots<i_{p}\right\} \subseteq\{-n, \ldots,-1,1, \ldots, p\}$ has the property that the number 


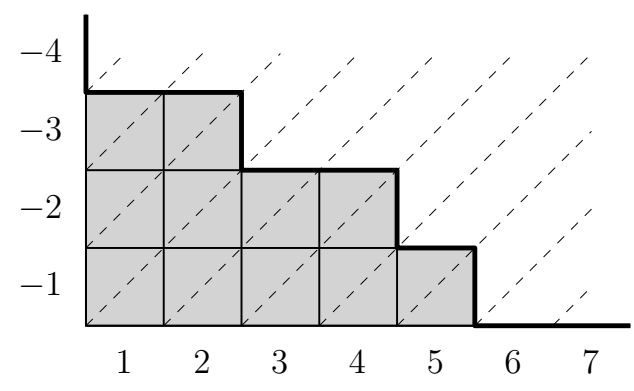

Figure 1. Lattice path and Young diagram corresponding to $I=$ $\{-3,-2,1,2,4,6,7,8, \ldots\}$.

of negative elements of $I$ equals $p$ minus the number of positive elements of $I$. Under the vertical map this property is preserved. Under the horizontal map, $x_{I}$ is identified with $x_{I^{\prime}}$ with $I^{\prime}=I \cup\{p+1\}$, and $I^{\prime}$ again has the property that its number of negative elements equals $p+1$ minus its number of positive elements.

Definition 3.1. The infinite wedge is defined as

$$
\bigwedge^{\frac{\infty}{2}} V_{\infty}:=\lim _{p, n} \bigwedge^{p} V_{n, p}=\bigcup_{p, n} \bigwedge^{p} V_{n, p}
$$

where the limit is taken of the directed system above. It comes with a standard basis consisting of elements $x_{I}=x_{i_{1}} \wedge x_{i_{2}} \wedge \cdots$ where $I=\left\{i_{1}<i_{2}<\ldots\right\} \subseteq-\mathbb{N} \cup \mathbb{N}$ has the property that $i_{k}=k$ for $k \gg 0$; this element is the image in $\bigwedge^{\frac{\infty}{2}} V_{\infty}$ of $x_{i_{1}} \wedge \cdots \wedge x_{i_{p}} \in \wedge^{p}\left(V_{n, p}\right)$ for any choice of $n \geq-i_{1}$ and of $p$ such that $i_{k}=k$ for $k \geq p$.

In fact, in the existing literature on the infinite wedge, this limit is called the charge zero part of the infinite wedge. The full infinite wedge then arises by allowing $k-i_{k}$ to be any constant for $k \gg 0$, and this constant is called the charge of $x_{I}$. We will restrict ourselves to the charge-zero part.

The basis vectors $x_{I}$ of $\bigwedge^{\frac{\infty}{2}} V_{\infty}$ are in one-to-one correspondence with the set of all Young diagrams, and this will be a useful visual aid later on. This correspondence is well known; see for instance the discussion of Maya diagrams and partitions in RZ13. To find the Young diagram corresponding to $I=\left\{i_{1}<i_{2}<\ldots\right\}$, proceed as follows. Draw the first quadrant $\mathbb{R}_{\geq 0}^{2}$ with horizontal axis subdivided into unit intervals labelled by $\mathbb{N}$ and vertical axis subdivided into unit intervals labelled by $-\mathbb{N}$. Subdivide the quadrant into diagonal strips labelled by $-\mathbb{N} \cup \mathbb{N}$ running from the corresponding (horizontal or vertical) intervals in northeasterly direction. Now draw the lattice path that starts high north on the vertical axis and goes south in a strip corresponding to an $i \notin I$ and east in a strip corresponding to an $i \in I$. The fact that $i_{k}=k$ for $k \gg 0$ ensures that the path ends up on the horizontal axis. The region below the lattice path is a Young diagram, which uniquely determines the lattice path and the set $I$. For an example see Figure 1.

The basis vectors $x_{I}$ have a natural partial order defined by $x_{I} \preceq x_{J}$ if and only if $i_{k} \leq j_{k}$ for all $k$. This is equivalent to the condition that the Young diagram corresponding to $I$ contains the Young diagram corresponding to $J$. The unique largest element has $I=\{1,2,3, \ldots\}$, and the partial order does not have infinite 
strictly increasing chains. In fact, a much stronger statement holds: the opposite partial order is a well-partial-order on the variables $x_{I}$ (and a similar statement holds for higher-dimensional partitions; see, e.g., Mac01]), but we will not need this stronger statement.

Despite its apparent dependence on the choice of coordinates, the infinite wedge has a large symmetry group acting on it. Indeed, denote $G_{n, p}:=\operatorname{GL}\left(V_{n, p}\right)$ and embed $G_{n, p}$ into $G_{n+1, p}$ and $G_{n, p+1}$ by fixing the standard basis vector $x_{-(n+1)}$ and $x_{p+1}$, respectively. Each of the two arrows emanating from $\bigwedge^{p} V_{n, p}$ is $G_{n, p^{-}}$ equivariant. As a consequence, the group $G_{\infty}=\bigcup_{n, p} G_{n, p}$ acts on $\bigwedge^{\frac{\infty}{2}} V_{\infty}$. More explicitly, if an element $g \in G_{n^{\prime}, p^{\prime}}$ is to act on an element $\omega \in \bigwedge^{p} V_{n^{\prime \prime}, p^{\prime \prime}}$, then one sets $n:=\max \left\{n^{\prime}, n^{\prime \prime}\right\}, p:=\max \left\{p^{\prime}, p^{\prime \prime}\right\}$, sees $g$ as an element of $G_{n, p}$ and $\omega$ as an element in $\Lambda^{p} V_{n, p}$, and performs the action there.

Example 3.2. We note two consequences of the $G_{\infty}$-action on $\bigwedge^{\frac{\infty}{2}} V_{\infty}$ that will become important later on. First, embedding the symmetric group $S_{n+p}$ by means of permutation matrices into $G_{n, p}$, we find that the group of all finitary permutations of $-\mathbb{N} \cup \mathbb{N}$, i.e., those that fix all but a finite number of integers, acts on $\bigwedge^{\frac{\infty}{2}} V_{\infty}$. A finitary permutation $\pi$ sends the basis vector $x_{I}$ to $\pm x_{\pi(I)}$, where the sign depends on the number of pairs $i, j \in I$ with $i<j$ but $\pi(i)>\pi(j)$. All signed basis vectors are contained in a single orbit under finitary permutations.

Second, the action of $G_{\infty}$ induces an action of its Lie algebra by taking derivatives. This Lie algebra is spanned by the derivations $\partial_{k l}:=x_{k} \cdot \frac{\partial}{\partial x_{l}}$ as $k, l$ vary over $-\mathbb{N} \cup \mathbb{N}$. The action of this derivation on a basis vector $x_{I}$ is obtained by writing $x_{I}=x_{i_{1}} \wedge x_{i_{2}} \wedge \cdots$ and formally applying Leibniz' rule. In other words,

$$
\partial_{k l} x_{I}= \begin{cases}x_{I} & \text { if } k=l \text { and } k \in I, \\ \pm x_{I \backslash\{l\} \cup\{k\}} & \text { if } k \notin I \text { and } l \in I, \text { and } \\ 0 & \text { otherwise, }\end{cases}
$$

with sign determined by the number of elements of $I$ strictly between $k$ and $l$. $\diamond$

Remark 3.3. The symmetric algebra generated by the infinite wedge has an action of $G_{\infty}$ by automorphisms. Since $G_{\infty}$ is isomorphic to the infinite general linear group, one might think that this symmetric algebra is a twisted commutative algebra (tca) in the sense of [SS12a, SS12b]. But this is not the case, since $\wedge^{\frac{\infty}{2}} V_{\infty}$ is not a subquotient of any finite tensor power of the countably-dimensional standard representation of the infinite general linear group. If one restricts the attention to those $x_{I}$ with $I \supseteq\{p, p+1, \ldots\}$ for some fixed $p \in \mathbb{N}$, acted on by the stabiliser in $G_{\infty}$ of all $x_{i}$ for $i \geq p$, then one does obtain a tca. However, such a tca is too small for our purposes. For instance, it does not allow for proving statements about all Grassmannians $\operatorname{Gr}(p, V)$ with both $p$ and $V$ varying.

In the following sections, we will be concerned with the dual infinite wedge $\left(\bigwedge^{\frac{\infty}{2}} V_{\infty}\right)^{*}$. This uncountably-dimensional vector space arises as the projective limit $\lim _{\longleftarrow} \bigwedge^{p} V_{n, p}^{*}$ of the diagram obtained from Diagram (2) by taking duals of all $\overleftarrow{n, p}$ 
arrows:

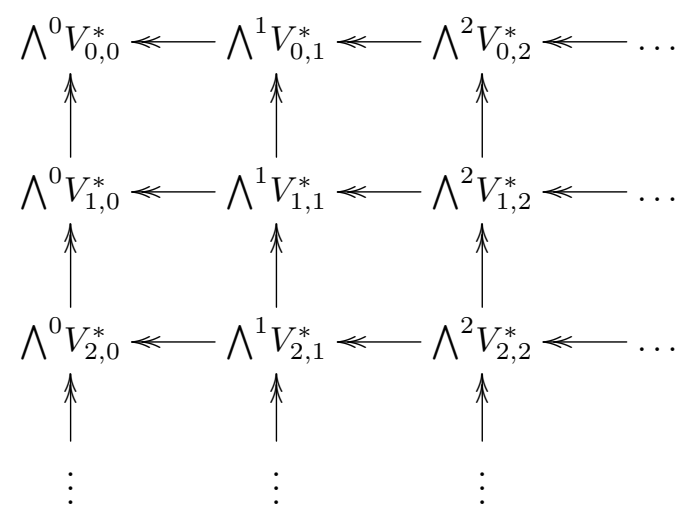

The symmetric algebra generated by $\bigwedge^{\frac{\infty}{2}} V_{\infty}$, which is the polynomial ring in the $x_{I}$, serves as coordinate ring of the dual infinite wedge. The dual infinite wedge carries the Zariski topology, in which the closed subsets are those characterised by the vanishing of a collection of polynomials in the $x_{I}$.

We will also need arrows going in the opposite direction. For this, denote the basis of $V_{n, p}^{*}$ dual to the standard basis by $e_{-n}, \ldots, e_{-1}, e_{1}, \ldots, e_{p}$. Take the $p$-th exterior power of the embedding $V_{n, p}^{*} \rightarrow V_{n+1, p}^{*}, e_{i} \mapsto e_{i}$ as vertical maps and the map

$$
\bigwedge^{p} V_{n, p}^{*} \rightarrow \bigwedge^{p+1} V_{n, p+1}^{*}, \quad \omega \mapsto \omega \wedge e_{p+1}
$$

as horizontal map. These maps are right inverses (sections) of the corresponding projections in Diagram (3), and they fit into the commutative diagram

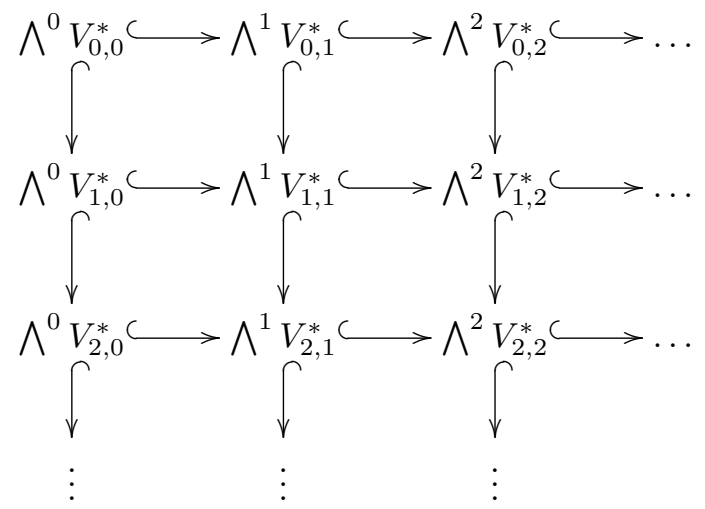

In particular, this diagram allows us to lift an element of $\bigwedge^{p} V_{n, p}^{*}$ to an element in the dual infinite wedge. We will return to this fact in Section 7 .

\section{The limit of A PlüCKer VARIEty}

Let $\mathbf{X}$ be a Plücker variety, and evaluate $\mathrm{X}_{n, p}:=\mathbf{X}_{p}\left(V_{n, p}^{*}\right)$. By the Plücker variety axioms, the embedded variety $\mathrm{X}_{n, p} \subseteq \bigwedge^{p} V_{n, p}^{*}$ is the image of the embedded variety $\mathbf{X}_{p}(V) \subseteq \bigwedge^{p} V$ for any $(n+p)$-dimensional vector space $V$ under any isomorphism $V \rightarrow V_{n, p}^{*}$. Hence, the $\mathrm{X}_{n, p}$ determine the Plücker variety and they are stable under $G_{n, p}=\mathrm{GL}\left(V_{n, p}\right)$. 
Next, the $X_{n, p}$ fit into two commutative diagrams

$(5)$

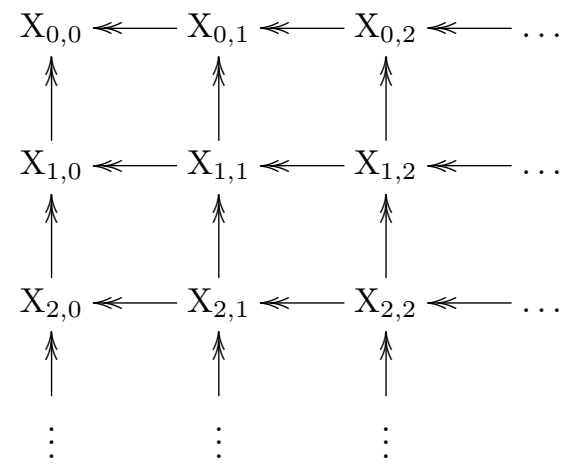

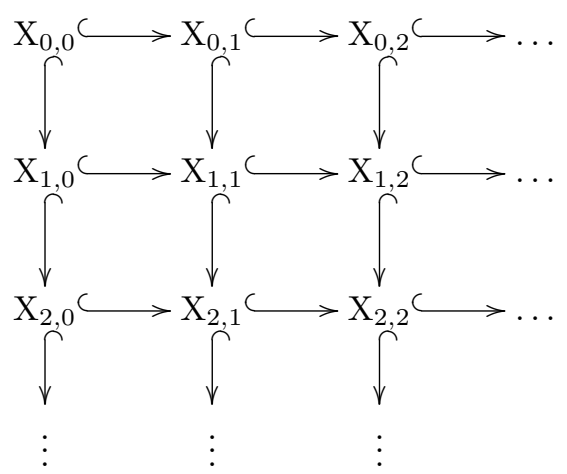

where the maps in the leftmost diagram are those from Diagram 3 and those in the rightmost diagram are those from Diagram 4. The horizontal maps preserve instances of Plücker varieties because of Lemmas 2.4 and 2.3, respectively. The vertical maps preserve instances by Definition 2.1 (2).

We denote $\mathrm{X}_{\infty}:=\lim X_{n, p} \subseteq\left(\bigwedge^{\frac{\infty}{2}} V_{\infty}\right)^{*}$, which we call the limit of the Plücker variety $\mathbf{X}$. This is the subset of the dual infinite wedge consisting of all $\omega$ with the property that for each $n, p$ the image of $\omega$ in $\bigwedge^{p} V_{n, p}^{*}$ lies in $\mathrm{X}_{n, p}$. Equivalently, it is the zero set of the union of all ideals of the $\mathrm{X}_{n, p}$ in the polynomial ring in the variables $x_{I} \in \bigwedge^{\frac{\infty}{2}} V_{\infty}$. Since each $\mathrm{X}_{n, p}$ is $G_{n, p}$-stable, $\mathrm{X}_{\infty}$ is a $G_{\infty}$-stable, closed subset of $\left(\bigwedge^{\frac{\infty}{2}} V_{\infty}\right)^{*}$.

Example 4.1. For the Grassmannian $\mathbf{X}_{p}(V):=\operatorname{Gr}(p, V)$, the limit $\mathrm{X}_{\infty}$ is (the charge zero part of) Sato's Grassmannian [SS83, SW85]; for a more algebraic treatment see VMP98. It is the common zero set in $\left(\bigwedge^{\frac{\infty}{2}} V_{\infty}\right)^{*}$ of all polynomials of the form

$$
\sum_{k=1}^{\infty}(-1)^{k} x_{I \backslash\left\{i_{k}\right\}}\left(x_{i_{k}} \wedge x_{J}\right)
$$

where $I=\left\{i_{1}<i_{2}<\ldots\right\}$ is a subset of $-\mathbb{N} \cup \mathbb{N}$ with $k-i_{k}=1$ for $k \gg 0$ (charge 1) and $J=\left\{j_{1}<j_{2}<\ldots\right\}$ is a subset with $k-j_{k}=-1$ for $k \gg 0$ (charge -1) and where $x_{i_{k}} \wedge x_{J}$ equals $\pm x_{J \cup\left\{i_{k}\right\}}$ if $i_{k} \notin J$ (sign depending on the parity of the position of $i_{k}$ among the $j_{l}$ ) and zero otherwise. Note that this is, indeed, a polynomial, since $i_{k} \in J$ for $k \gg 0$. In characteristic zero, these Plücker relations generate the ideal of $\mathrm{X}_{\infty}$; for positive characteristic see [Abe80, BC03. The simplest Plücker relation comes from $\mathbf{X}_{2}\left(V_{2,2}\right)$ and reads

$$
x_{-2,-1,3, \ldots} x_{1,2,3, \ldots}-x_{-2,1,3, \ldots} x_{-1,2,3, \ldots}+x_{-2,2,3, \ldots} x_{-1,1,3, \ldots}
$$

or, in the Young diagram notation:
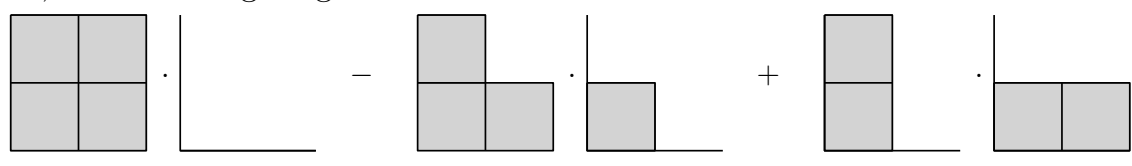

\section{Pfaffians on the dual infinite Wedge}

To test whether an element $\omega$ of $\bigwedge^{2} V$ has rank less than $r$, one can use Pfaffians. We recall the definition. 
Definition 5.1. Let $A=\left(a_{i, j}\right)_{i, j=1}^{2 r}$ be a skew-symmetric matrix. Then the Pfaffian of $A$ is defined as $\operatorname{Pf}(A)=\frac{1}{2^{r} r !} \sum_{\sigma \in \operatorname{Sym}_{2 r}} \operatorname{sign}(\sigma) \prod_{i=1}^{r} a_{\sigma(2 i-1) \sigma(2 i)}$. Its square is the determinant of $A$.

If we write the Pfaffian of $A$ as a polynomial in $\mathbb{Q}\left[a_{i, j}\right]$, then its coefficients are integers. In fact, if $\sigma \in \mathrm{Sym}_{2 r}$, then the monomial $\prod_{i=1}^{r} a_{\sigma(2 i-1) \sigma(2 i)}$ has coefficient $\operatorname{sign}(\sigma)$ in $\operatorname{Pf}(A)$. Hence the definition of $\mathrm{Pf}$ makes sense over fields of positive characteristic, as well.

For a choice of linearly independent $x_{1}, \ldots, x_{2 r} \in V^{*}$ we can form the matrix $A=\left(x_{i} \wedge x_{j}\right)_{i j}$ of linear functions on $\bigwedge^{2} V$, and its $\operatorname{Pfaffian} \operatorname{Pf}(A)$ is a degree$r$ polynomial function on $\Lambda^{2}(A)$. A polynomial obtained like this is called an order-r sub-Pfaffian on $\bigwedge^{2} V$. The square of an order- $r$ sub-Pfaffian is an order- $2 r$ sub-determinant on $\bigwedge^{2} V$, and $\omega$ has rank less than $r$ if and only if all order- $r$ sub-Pfaffians vanish on it. In other words, the variety $Y^{r}(V)$ is the common zero set of all $(r+1)$-th sub-Pfaffians on $\bigwedge^{2} V$. Note that the $(r+1)$-th sub-Pfaffians form a single $\mathrm{GL}(V)$-orbit.

Returning to the $V_{n, 2}$ from the definition of the infinite wedge, observe that $\bigwedge^{2} V_{n, 2}^{*}$ has coordinates $x_{i, j}:=x_{i} \wedge x_{j}=-x_{i, j}$ with $i, j \in\{-n, \ldots,-2,-1,1,2\}$. We take $n=2 r$ and define

$$
\operatorname{Pf}_{r+1}:=\operatorname{Pf}\left(\left(x_{i, j}\right)_{i, j \in\{-2 r, \ldots,-2,-1,1,2\}}\right) .
$$

This is a polynomial function on $\bigwedge^{2} V_{n, 2}^{*}$ and hence, by Diagram (2), on the dual infinite wedge. These specific Pfaffians satisfy the following recursion that will be exploited in Section 6 .

Lemma 5.2. Assume that $r+1 \geq 2$. Among the variables $x_{I}$ appearing in the Pfaffian $\mathrm{Pf}_{r+1}$, there is a unique $\preceq$-minimal one, namely, $x_{-2 r,-2 r+1}=x_{-2 r,-2 r+1,3,4, \ldots}$. Moreover, we have the recursion

$$
\operatorname{Pf}_{r+1}=x_{-2 r,-2 r+1} \cdot \mathrm{Pf}_{r}+Q_{r+1}
$$

where $Q_{r+1}$ is a polynomial of degree $r+1$ in variables $\succ x_{-2 r,-2 r+1,3,4, \ldots}$.

Proof. The first statement is obvious, since all variables are of the form $x_{i} \wedge x_{j}$ with $-2 r \leq i<j \leq 2$ and therefore $-2 r \leq i$ and $-2 r+1 \leq j$. For the second statement, note that any monomial occurring in $\operatorname{Pf}_{r+1}$ containing $x_{-2 r,-2 r+1}$ is of the form $x_{-2 r,-2 r+1} M_{r}$ with $M_{r}$ a monomial occurring in $\mathrm{Pf}_{r}$, and the coefficient of $M_{r}$ in $\mathrm{Pf}_{r}$ is the coefficient of $x_{-2 r,-2 r+1} M_{r}$ in $\mathrm{Pf}_{r+1}$ (namely, it is the sign of the permutation used to form $M_{r}$ ). This shows that the coefficient of $x_{-2 r,-2 r+1}$ in $\mathrm{Pf}_{r+1}$ is, indeed, $\mathrm{Pf}_{r}$.

Dually, the pullback of $\mathrm{Pf}_{r+1}$ under a Hodge dual $\bigwedge^{2 r} V_{2 r, 2} \rightarrow \bigwedge^{2} V_{2 r, 2}^{*}$ is the equation for the hypersurface $Y^{r, \star}\left(V_{2 r, 2}\right)$. Pulling back further along the exterior power of an isomorphism $V_{2,2 r}^{*} \rightarrow V_{2 r, 2}$, we find the dual Pfaffian $\mathrm{Pf}_{r+1}^{\star}$, which is the polynomial function on $\bigwedge^{2 r} V_{2,2 r}^{*}$ whose vanishing characterises elements that are not of full rank. Again, we can regard $\mathrm{Pf}_{r+1}^{\star}$ as a polynomial on the dual infinite wedge. If we choose the scaling correctly, then we have the following analogue of the previous lemma.

Lemma 5.3. Among the variables $x_{I}$ appearing in the Pfaffian $\mathrm{Pf}_{r+1}^{\star}$, there is a unique $\preceq$-minimal one, namely, $x_{-2,-1,1,2, \ldots, 2 r-2}=x_{-2,-1,1,2, \ldots, 2 r-2,2 r+1,2 r+2, \ldots}$. 
Moreover, we have the recursion

$$
\mathrm{Pf}_{r+1}^{\star}=x_{-2,-1,1,2, \ldots, 2 r-2} \cdot \mathrm{Pf}_{r}^{\star}+Q_{r+1}^{\star}
$$

where $Q_{r+1}^{\star}$ is a polynomial in variables $\succ x_{-2,-1,1,2, \ldots, 2 r-2,2 r+1,2 r+2, \ldots}$.

For later use, we observe that the Young diagram of the smallest variable in $\mathrm{Pf}_{r+1}$ is a rectangle of width 2 and height $2 r$, while the Young diagram of the smallest variable in $\mathrm{Pf}_{r+1}^{\star}$ is a rectangle of height 2 and width $2 r$ :

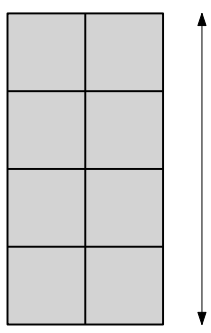

$2 r$

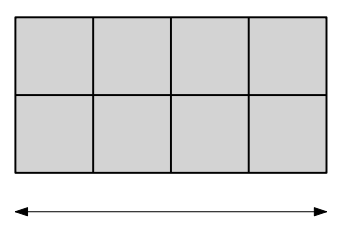

$2 r$

All other variables have Young diagrams strictly contained in these rectangles.

Example 5.4. For $r=1$ we have

$$
\mathrm{Pf}_{1}=x_{1,2}=\mathrm{Pf}_{1}^{\star}
$$

For $r=2$ we have

$$
\mathrm{Pf}_{2}=x_{-2,-1} x_{1,2}-x_{-2,1} x_{-1,2}+x_{-2,2} x_{-1,1}=\mathrm{Pf}_{2}^{\star} .
$$

However, for $r=3$ we have

$$
\begin{aligned}
\mathrm{Pf}_{3}= & x_{-4,-3} x_{-2,-1} x_{1,2}-x_{-4,-3} x_{-2,1} x_{-1,2}+\ldots+x_{-4,2} x_{-3,1} x_{-2,-1} \text { and } \\
\mathrm{Pf}_{3}^{\star}= & x_{-2,-1,1,2} x_{-2,-1,3,4} x_{1,2,3,4}-x_{-2,-1,1,2} x_{-2,1,3,4} x_{-1,2,3,4} \\
& +\ldots+x_{-1,1,2,3} x_{-2,1,2,4} x_{-2,-1,3,4}
\end{aligned}
$$

These polynomials are essentially different even when both are viewed as polynomials on $\bigwedge^{4} V_{4,4}^{*}$, which is the smallest $\bigwedge^{p} V_{n, p}^{*}$ on which both are defined.

\section{Equivariant Noetherianity of Pfaffian varieties}

This section contains the heart of our proof of Theorems 1.1 and 1.3 It deals with the following closed subsets of the dual infinite wedge.

Definition 6.1. For $r, s \in \mathbb{Z}_{\geq 0}$, we define $Y_{\infty}^{r, s}$ as

$$
Y_{\infty}^{r, s}:=\left\{\omega \in\left(\bigwedge^{\frac{\infty}{2}} V_{\infty}\right)^{*} \mid \forall g \in G_{\infty}: \operatorname{Pf}_{r+1}(g \omega)=\operatorname{Pf}_{s+1}^{\star}(g \omega)=0\right\} .
$$

We call $Y_{\infty}^{r, s}$ a Pfaffian variety.

By construction, $Y_{\infty}^{r, s}$ is a closed, $G_{\infty}$-stable subset of the dual infinite wedge. The main result of this section is as follows.

Theorem 6.2. For all $r, s \in \mathbb{Z}_{\geq 0}$, the variety $Y_{\infty}^{r, s}$ is $G_{\infty}$-Noetherian. In other words, every $G_{\infty}$-stable closed subset of $Y_{\infty}^{r, s}$ is cut out by finitely many $G_{\infty}$-orbits of polynomial equations.

We will need the following lemma on the complement of Pfaffian varieties. 
Lemma 6.3. Let $\omega \in\left(\bigwedge^{\frac{\infty}{2}} V_{\infty}\right)^{*}$ and suppose that there exist $g_{1}, g_{2} \in G_{\infty}$ such that $\mathrm{Pf}_{r}\left(g_{1} \omega\right) \neq 0$ and $\operatorname{Pf}_{s}^{\star}\left(g_{2} \omega\right) \neq 0$. Then there exists a $g \in G_{\infty}$ such that both $\operatorname{Pf}_{r}(g \omega) \neq 0$ and $\operatorname{Pf}_{s}^{\star}(g \omega) \neq 0$.

Proof. Consider $g=\lambda g_{1}+\mu g_{2}$, where $\lambda, \mu \in K$. Expand $\operatorname{Pf}_{r}(g \omega)$ as a formal polynomial in $\lambda, \mu$. Observe that the coefficient at $\lambda^{r}$ is $\operatorname{Pf}_{r}\left(g_{1} \omega\right) \neq 0$. Similarly, observe that the coefficient at $\mu^{s}$ of $\operatorname{Pf}_{s}^{\star}(g \omega)$ is $\operatorname{Pf}_{s}^{\star}\left(g_{2} \omega\right) \neq 0$. So the formal polynomials obtained are both non-zero, and hence the set

$$
\left\{(\lambda, \mu) \in K^{2}: g \notin G_{\infty} \vee \operatorname{Pf}_{r}(g \omega)=0 \vee \operatorname{Pf}_{s}^{\star}(g \omega)=0\right\}
$$

is a proper Zariski-closed subset of $K^{2}$ (using the fact that $K$ is infinite). So there exist $\lambda, \mu \in K$ such that $g \in G_{\infty}$ and $\operatorname{Pf}_{r}(g \omega) \neq 0$ and $\operatorname{Pf}_{s}^{\star}(g \omega) \neq 0$, and $g \in G_{\infty}$.

To prove Theorem 6.2 we proceed by induction. First, if either $r=0$ or $s=0$, then the Pfaffian $\mathrm{Pf}_{1}=x_{1,2}=\mathrm{Pf}_{1}^{\star}$ vanishes on $Y_{\infty}^{r, s}$. But then so do all polynomials in the $G_{\infty}$-orbit of $x_{1,2}=x_{1,2,3, \ldots}$, which contains all $x_{I}$. Hence then $Y^{r, s}$ consists of the single point 0 and is certainly equivariantly Noetherian. In the induction step, we may therefore assume that $r, s \geq 1$. We then write

$$
Y_{\infty}^{r, s}=Y_{\infty}^{r-1, s} \cup Y_{\infty}^{r, s-1} \cup Z^{\prime}
$$

where $Z^{\prime}$ is the subset of $\omega \in Y_{\infty}^{r, s}$ for which there exist $g_{1}, g_{2} \in G_{\infty}$ such that $\operatorname{Pf}_{r}\left(g_{1} \omega\right), \mathrm{Pf}_{s}^{\star}\left(g_{2} \omega\right)$ are both non-zero. By induction we know that the first two terms are $G_{\infty}$-Noetherian, so it suffices to prove that $Z^{\prime}$ is. By the previous lemma, we have $Z^{\prime}=G_{\infty} Z$ where

$$
Z:=\left\{\omega \in Y_{\infty}^{r, s} \mid \operatorname{Pf}_{r}(\omega) \neq 0 \text { and } \operatorname{Pf}_{s}^{\star}(\omega) \neq 0\right\} .
$$

We now set out to prove that $Z$ is equivariantly Noetherian under a suitable subgroup $H$ of $G_{\infty}$. To define this group, let $G_{-\infty,-2 r+1}$ denote the subgroup of $G_{\infty}$ of all maps that fix all $x_{i} \in V_{\infty}$ with $i \geq-2 r+2$. By Lemma 5.2 with $r$ replaced by $r-1$, this group fixes $\operatorname{Pf}_{r}$ (and also $\mathrm{Pf}_{s}^{\star}$ by Lemma 5.3). Similarly, let $G_{2 s-1, \infty}$ denote the group of all matrices that fix all $x_{i}$ with $i \leq 2 s-2$. By Lemma 5.3 with $r$ replaced by $s-1$, each variable $x_{I}$ in $\operatorname{Pf}_{s}^{\star}$ has $\{2 s-1,2 s, 2 s+1, \ldots\} \subseteq I$, so that an element $g \in G_{2 s-1, \infty}$ scales $x_{I}$ by $\operatorname{det}(g)$ and hence $\operatorname{Pf}_{s}^{\star}$ by $\operatorname{det}(g)^{s}$ (and scales $\mathrm{Pf}_{r}$ by $\left.\operatorname{det}(g)^{r}\right)$. We conclude that the open subset $Z$ of $Y_{\infty}^{r, s}$ is stable under the group

$$
H:=G_{-\infty,-2 r+1} \times G_{2 s-1, \infty} \subseteq G_{\infty} .
$$

To prove that $Z$ is $H$-Noetherian, we will embed it $H$-equivariantly into a space of the type in Theorem 1.5. To do so, we will use the equations of $Y_{\infty}^{r, s}$ to show that a point $\omega \in Z$ is in fact determined uniquely by a subset of its coordinates $x_{I}(\omega)$. These are the coordinates with $I$ as in the following definition.

Definition 6.4. Let $I \subseteq-\mathbb{N} \cup \mathbb{N}$ be a set of charge 0. We call $I$ (or $x_{I}$ ) good if both $I \cap \mathbb{Z}_{\leq-2 r+1}$ and $I^{c} \cap \mathbb{Z}_{>2 s-1}$ have cardinality at most 1 .

A good $I$ corresponds to a lattice path that goes east at most once to the north of the diagonal strip corresponding to $-2 r+2$ and south at most once to the east of the diagonal strip corresponding to $2 s-2$, see Figure 2 .

We let $\left(\bigwedge^{\frac{\infty}{2}} V_{\infty}\right)_{g}$ be the subspace of $\bigwedge^{\frac{\infty}{2}} V_{\infty}$ spanned by the good coordinates $x_{I}$, and let $\left(\bigwedge^{\frac{\infty}{2}} V_{\infty}\right)_{g}^{*}$ be its dual. Observe that $H$ acts on these spaces, and that the natural projection $\left(\bigwedge^{\frac{\infty}{2}} V_{\infty}\right)^{*} \rightarrow\left(\bigwedge^{\frac{\infty}{2}} V_{\infty}\right)_{g}^{*}$ is $H$-equivariant. 


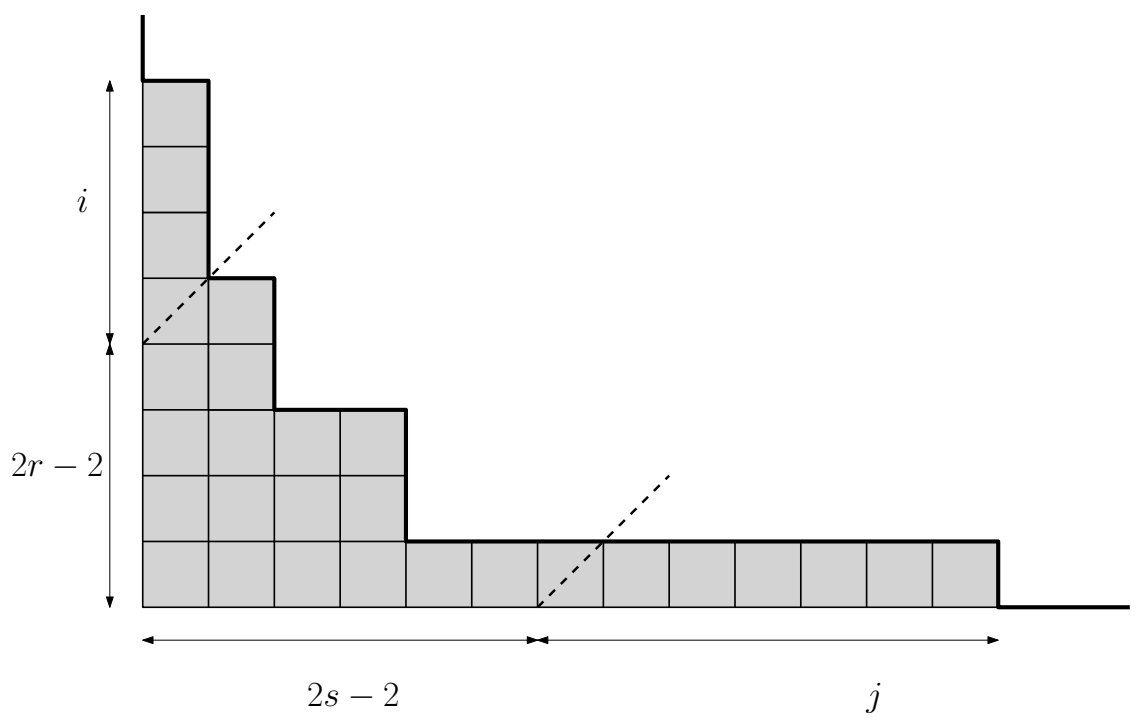

Figure 2. The lattice path corresponding to a good $I$.

Lemma 6.5. The topological space $\left(\bigwedge^{\frac{\infty}{2}} V_{\infty}\right)_{g}^{*}$ with the Zariski topology is $H$ Noetherian.

Proof. A coordinate $x_{I}$ on $\left(\bigwedge^{\frac{\infty}{2}} V_{\infty}\right)_{g}^{*}$ can be one of four possible types, depending on $\left|I \cap \mathbb{Z}_{<-2 r+2}\right| \in\{0,1\}$ and $\left|I^{c} \cap \mathbb{Z}_{>2 s-2}\right| \in\{0,1\}$. The coordinates with $\mid I \cap$ $\mathbb{Z}_{<-2 r+2}|=| I^{c} \cap \mathbb{Z}_{>2 s-2} \mid=0$ form a finite set, say of size $d$. The coordinates with $\left|I \cap \mathbb{Z}_{<-2 r+2}\right|=1$ and $\left|I^{c} \cap \mathbb{Z}_{>2 s-2}\right|=0$ can be organized in finitely many, say $n$, columns with row index equal to the unique element of $I \cap \mathbb{Z}_{<-2 r+2}$. These columns are acted upon by $G_{-\infty,-2 r+1}$. Similarly, the coordinates with $\left|I \cap \mathbb{Z}_{<-2 r+2}\right|=0$ and $\left|I^{c} \cap \mathbb{Z}_{>2 s-2}\right|=1$ can be organized in finitely many, say $m$, rows, acted upon by $G_{2 s-1, \infty}$. Finally, the coordinates with $\left|I \cap \mathbb{Z}_{<-2 r+2}\right|=\left|I^{c} \cap \mathbb{Z}_{>2 s-2}\right|=1$ can be organized in finitely many, say $p$, infinite-by-infinite matrices, on which $G_{-\infty,-2 r+1}$ acts by row operations, and on which $G_{2 s-1, \infty}$ acts by column operations. The row and column index of the good $x_{I}$ in Figure 2 for instance, equals $-2 r+2-i$ and $2 s-2+j$.

Thus, after relabelling the column and row indices to take values in $\mathbb{N}$ (so replacing $-2 r+2-i$ by $i$ and $2 s-2+j$ by $j$ ), an element of $\left(\bigwedge^{\frac{\infty}{2}} V_{\infty}\right)_{g}^{*}$ can be seen as a tuple of a vector in $K^{d}$, an element of $K^{\mathbb{N} \times n}$, and element of $K^{m \times \mathbb{N}}$, and an element of $\left(K^{\mathbb{N} \times \mathbb{N}}\right)^{p}$, and the action of $H$ corresponds to the diagonal action of $\mathrm{GL}_{\mathbb{N}} \times \mathrm{GL}_{\mathbb{N}}$ on this space. Now Theorem 1.5 implies the lemma.

Before we continue with the proof that $Z$ is $H$-Noetherian, we recall that the coordinates $x_{I}$ are partially ordered by $\preceq$, which corresponds to opposite containment of Young diagrams. This order is compatible with the "upper triangular" derivations $\partial_{k, l}, k<l$ from Example 3.2 in the following sense: first, if $\partial_{k, l} x_{I}$ is non-zero, then it equals $\pm X_{J}$ with $J \prec I$. Second, if also $\partial_{k, l} x_{K}= \pm X_{L}$ is non-zero and if $I \prec K$, then $J \prec L$.

We now consider the projection $Z \rightarrow\left(\bigwedge^{\frac{\infty}{2}} V_{\infty}\right)_{g}^{*}$ that takes a point $\omega$ and forgets all its coordinates except for the good ones. We claim that this map is injective, 
and in fact a closed embedding into the open subset of $\left(\bigwedge^{\frac{\infty}{2}} V_{\infty}\right)_{g}^{*}$ where both $\operatorname{Pf}_{r}$ and $\mathrm{Pf}_{s}^{\star}$ are non-zero. For this it suffices to show that, on $Z$, each coordinate $x_{I}$ can be expressed as a rational function in the good coordinates, whose denominator only has factors $\mathrm{Pf}_{r}$ and $\mathrm{Pf}_{s}^{\star}$. If $I$ is good, then $x_{I}$ itself is such an expression. Now we proceed by induction relative to the partial order $\preceq$. So let $I$ be not good, and assume that for all $J \succ I$ such a rational expression exists for $x_{J}$. Since $I$ is not good, one of the following two cases applies.

First, suppose $\left|I \cap \mathbb{Z}_{\leq-2 r+1}\right|>1$. Then $I \preceq\{-2 r,-2 r+1,3,4, \ldots\}$. On $Y_{\infty}^{r, s}$ we have

$$
0=\operatorname{Pf}_{r+1}=x_{-2 r,-2 r+1,3,4, \ldots} \operatorname{Pf}_{r}+Q_{r+1}
$$

by Lemma 5.2, where both $\operatorname{Pf}_{r}$ and $Q_{r+1}$ contain only variables $x_{J}$ with $J \succ$ $\{-2 r,-2 r+1,3,4, \ldots\}$. Write $I=\left\{i_{1}<i_{2}<\ldots\right\}$ and let $k \geq 2$ be the maximal index for which $i_{k}<k$. Consider the differential operator

$$
D:=\partial_{i_{k}, k} \circ \partial_{i_{k-1}, k-1} \circ \cdots \circ \partial_{i_{3}, 3} \circ \partial_{i_{2},-2 r+1} \circ \partial_{i_{1},-2 r}
$$

which is chosen such that $D x_{-2 r,-2 r+1,3,4, \ldots}=x_{I}$. We stress the order: first $\partial_{i_{1},-2 r}$ has the effect of replacing $-2 r$ by $i_{1}$, then $-2 r+1$ is replaced by $i_{2}$, etc. Since $Y_{\infty}^{r, s}$ is $G_{\infty}$-stable, the ideal of polynomials vanishing on it is stable under $D$. Applying $D$ to the equation above, and using the Leibniz rule, we find that

$$
0=x_{I} \operatorname{Pf}_{r}+P+D Q_{r+1}
$$

holds on $Y_{\infty}^{r, s}$, where $P$ is obtained from the product $x_{-2 r,-2 r+1,3,4, \ldots} \cdot \mathrm{Pf}_{r}$ by letting at least one of the factors of $D$ act on $\mathrm{Pf}_{r}$ and the remaining factors act on $x_{-2 r,-2 r+1,3,4, \ldots}$. By the discussion above, the variables appearing in $P$ and in $D Q_{r+1}$ are all strictly greater than $x_{I}$, so for those variables a rational expression exists as desired by the induction hypothesis. But then also for

$$
x_{I}=\left(-P-D Q_{r+1}\right) / \mathrm{Pf}_{r}
$$

such an expression exists.

Second, otherwise we have $\left|I^{c} \cap \mathbb{Z}_{\geq 2 s-1}\right|>1$. Then we have

$$
I \preceq\{-2,-1,1,2, \ldots, 2 s-2,2 s+1,2 s+2, \ldots\} .
$$

On $Y_{\infty}^{r, s}$ we have

$$
0=\mathrm{Pf}_{s+1}^{\star}=x_{-2,-1,1,2, \ldots, 2 s-2} \cdot \mathrm{Pf}_{s}^{\star}+Q_{s+1}^{\star}
$$

where all variables in $\mathrm{Pf}_{s}^{\star}$ and $Q_{s+1}^{\star}$ are strictly larger than $x_{-2,-1,1,2, \ldots, 2 s-2}$. Again, write $I=\left\{i_{1}<i_{2}<\ldots\right\}$, let $k \geq 2 s$ be maximal with $i_{k} \neq k$ and apply the differential operator

$$
D=\partial_{i_{k}, k} \circ \cdots \circ \partial_{i_{2 s+1}, 2 s+1} \circ \partial_{i_{2 s}, 2 s-2} \cdots \circ \partial_{i_{3}, 1} \circ \partial_{i_{2},-1} \circ \partial_{i_{1},-2}
$$

to the equation above to find an expression for $x_{I}$.

We conclude that the topological space $Z$ is isomorphic to an $H$-stable locally closed subset of $\left(\bigwedge^{\frac{\infty}{2}} V_{\infty}\right)_{g}^{*}$ with the induced topology. Since the latter space is $H$-Noetherian, so is $Z$. A basic observation on equivariant Noetherianity is that when the $H$-Noetherian space $Z$ is smeared out by the larger group $G_{\infty} \supseteq H$, then the resulting topological space is $G_{\infty} Z \subseteq\left(\bigwedge^{\frac{\infty}{2}} V_{\infty}\right)^{*}$ is $G_{\infty}$-Noetherian (see [DK14, Lemma 5.4]). Moreover, the union of finitely many $G_{\infty}$-Noetherian spaces is $G_{\infty}$-Noetherian, hence in particular so is

$$
Y_{\infty}^{r, s}=Y_{\infty}^{r-1, s} \cup Y_{\infty}^{r, s-1} \cup G_{\infty} Z
$$


This concludes the proof of Theorem 6.2 given Theorem 1.5 the latter will be proved in Section 8. A direct consequence of that theorem is the following.

Corollary 6.6. Let $\mathbf{X}$ be a bounded Plücker variety. Then its limit $\mathrm{X}_{\infty}$ is defined in $\left(\bigwedge^{\frac{\infty}{2}} V_{\infty}\right)^{*}$ by the $G_{\infty}$-orbits of finitely many polynomial equations.

Proof. The limit $\mathrm{X}_{\infty}$ is a closed, $G_{\infty}$-stable subset of $Y_{\infty}^{r, r}$, where $r$ is the rank of the bounded Plücker variety. Since $Y_{\infty}^{r, r}$ is $G_{\infty}$-Noetherian, $\mathrm{X}_{\infty}$ is defined within $Y_{\infty}^{r, r}$ by the $G_{\infty}$-orbits of finitely many equations. Adding to these the single $G_{\infty} \operatorname{Pf}_{r}=$ $\mathrm{Pf}_{r}^{\star}$, the corollary follows.

\section{BACK TO FINITE-DIMENSIONAL INSTANCES}

We have now proved a fundamental result, Corollary 6.6, on the limit of a bounded Plücker variety $\mathbf{X}$. This is a projective limit of all instances of the Plücker variety, hence projects to all of them. To draw conclusions for these instances themselves, however, we need to be able to lift them back into the limit. For this we make use of Diagram (4). This diagram allows us to extend a single $\omega_{n_{1}, p_{1}} \in \bigwedge^{p_{1}} V_{n_{1}, p_{1}}^{*}$ to a point $\omega=\left(\omega_{n, p}\right)_{n, p \in \mathbb{Z}_{\geq 0}}$ in the dual infinite wedge. By the right-most diagram in (5), this point $\omega$ lies in $\mathrm{X}_{\infty}$ if and only if $\omega_{n_{1}, p_{1}}$ lies in $X_{n_{1}, p_{1}}$. We can now easily prove the main theorem.

Proof of the main theorem. By Corollary 6.6 there exist $n_{0}, p_{0}$ such that the $G_{\infty^{-}}$ orbits of the equations of $X_{n_{0}, p_{0}}$ define $\mathrm{X}_{\infty}$. Thus for arbitrary $n, p$ we can find polynomials $f_{1}, \ldots, f_{N}$ in the ideal of $X_{n_{0}, p_{0}}$ and group elements $g_{1}, \ldots, g_{N} \in G_{\infty}$ such that the $g_{i} f_{i}$, restricted to $\bigwedge^{p} V_{n, p}^{*}$ via Diagram 4 , define $X_{n, p}$ set-theoretically. Each of these equations $g_{i} f_{i}$ arises as a pullback of $f_{i}$ under a sequence of linear maps as in the definition of a Plücker variety.

This proof is slightly unsatisfactory in that we seem to have no control over the elements $g_{i}$. A priori, they may have to be chosen from $G_{n^{\prime}, p^{\prime}}$ with $n^{\prime}, p^{\prime}$ much larger than the relevant $n, n_{0}, p, p_{0}$. Our next goal is to show that this is not the case. After the $g_{i}$ are under control, also Theorem 1.3 follows.

From now on, we write $\pi_{n_{0}, p_{0}} \omega$ for the image in $\bigwedge^{p_{0}} V_{n_{0}, p_{0}}^{*}$ of a point $\omega$ in the dual infinite wedge. We will use the same notation when $\omega$ lies in some finite $\bigwedge^{p} V_{n, p}^{*}$. Then it is understood that $\omega$ is first lifted to the dual infinite wedge and then projected.

Lemma 7.1. Let $\mathbf{X}$ be a Plücker variety, $n, p \in \mathbb{Z}_{\geq 0}$, and $\omega \in \bigwedge^{p} V_{n, p}^{*}$. Let $n_{0}, p_{0}, n^{\prime}, p^{\prime} \in \mathbb{Z}_{\geq 0}$ with $n^{\prime} \geq \max \left\{n, n_{0}\right\}$ and $p^{\prime} \geq \max \left\{p, p_{0}\right\}$ and suppose that there exists a $g \in G_{n^{\prime}, p^{\prime}}$ such that $\pi_{n_{0}, p_{0}} g(\omega) \notin \mathrm{X}_{n_{0}, p_{0}}$. Then the following hold.

(1) If $p^{\prime}>p$ and $p^{\prime}>p_{0}$, then $\exists g^{\prime} \in G_{n^{\prime}, p^{\prime}-1}: \pi_{n_{0}, p_{0}} g^{\prime}(\omega) \notin \mathrm{X}_{n_{0}, p_{0}}$.

(2) If $p^{\prime}>p$ and $p^{\prime}=p_{0}$, then $\exists g^{\prime} \in G_{n^{\prime}, p^{\prime}-1}: \pi_{n_{0}, p_{0}-1} g(\omega) \notin \mathrm{X}_{n_{0}, p_{0}-1}$.

(3) If $n^{\prime}>n$ and $n^{\prime}>n_{0}$, then $\exists g^{\prime} \in G_{n^{\prime}-1, p^{\prime}}: \pi_{n_{0}, p_{0}} g(\omega) \notin \mathrm{X}_{n_{0}, p_{0}}$.

(4) If $n^{\prime}>n$ and $n^{\prime}=n_{0}$, then $\exists g^{\prime} \in G_{n^{\prime}-1, p^{\prime}}: \pi_{n_{0}-1, p_{0}} g^{\prime}(\omega) \notin \mathrm{X}_{n_{0}-1, p_{0}}$.

Proof. The first part and the third part of the lemma are dual to each other, and so are the second part and the fourth part. Therefore, it suffices to prove only the first two parts. Moreover, the condition $\pi_{n_{0}, p_{0}} g(\omega) \notin X_{n_{0}, p_{0}}$ holds for $g$ in a nonempty and open, hence dense, subset of $G_{n^{\prime}, p^{\prime}}$, so we may assume that $g$ from the statement of the lemma is sufficiently general. Recall that $V_{n^{\prime}, p^{\prime}}^{*}=\left\langle e_{i}\right\rangle_{-n^{\prime} \leq i \leq p^{\prime}, i \neq 0}$, with corresponding coordinates $x_{i}$. 
Suppose that $p^{\prime}>p$. We may assume that $x_{p^{\prime}}\left(g e_{p^{\prime}}\right) \neq 0$. We define the linear $\operatorname{map} g^{\prime}$ on $V_{n^{\prime}, p^{\prime}-1}^{*}$ by

$$
g^{\prime} v=g v-\frac{x_{p^{\prime}}(g v)}{x_{p^{\prime}}\left(g e_{p^{\prime}}\right)} g e_{p^{\prime}}, v \in V_{n^{\prime}, p^{\prime}-1}^{*} .
$$

In other words, $g^{\prime}$ equals the composition of $\left.g\right|_{V_{n^{\prime}, p^{\prime}-1}^{*}}: V_{n^{\prime}, p^{\prime}-1}^{*} \rightarrow V_{n^{\prime}, p^{\prime}}^{*}$ and the projection $V_{n^{\prime}, p^{\prime}}^{*} \rightarrow V_{n^{\prime}, p^{\prime}-1}^{*}$ along $g e_{p^{\prime}}$. We view $g^{\prime}$ as an element of $G_{n^{\prime}, p^{\prime}}$ by inclusion, i.e., fixing $e_{p^{\prime}}$. In $\bigwedge^{p^{\prime}} V_{n^{\prime}, p^{\prime}}^{*}$ we compute

$$
g^{\prime} \pi_{n^{\prime}, p^{\prime}-1}(\omega) \wedge g e_{p^{\prime}}=g \pi_{n^{\prime}, p^{\prime}-1}(\omega) \wedge g e_{p^{\prime}}=g\left(\pi_{n^{\prime}, p^{\prime}-1}(\omega) \wedge e_{p^{\prime}}\right)=g\left(\pi_{n^{\prime}, p^{\prime}}(\omega)\right) .
$$

Here the first equality follows from basic properties of alternating tensors and the last equality follows from $p^{\prime}>p$, which means that to go from $\omega$ to $\pi_{n^{\prime}, p^{\prime}} \omega$ one tensors with $p^{\prime}-p>0$ factors on the right, and then follows the inclusion $\bigwedge^{p^{\prime}} V_{n, p^{\prime}}^{*} \rightarrow \bigwedge^{p^{\prime}} V_{n^{\prime}, p^{\prime}}^{*}$. Contracting both sides with $x_{p^{\prime}}$ yields

$$
x_{p^{\prime}}\left(g e_{p^{\prime}}\right) \cdot g^{\prime} \pi_{n^{\prime}, p^{\prime}-1}(\omega)=\pi_{n^{\prime}, p^{\prime}-1}(g \omega) .
$$

Now if $p^{\prime}>p_{0}$, then we can further down and find

$$
\pi_{n_{0}, p_{0}} g^{\prime}(\omega)=\frac{1}{x_{p^{\prime}}\left(g e_{p^{\prime}}\right)} \pi_{n_{0}, p_{0}} g(\omega) \notin \mathrm{X}_{n_{0}, p_{0}} .
$$

If $p^{\prime}=p_{0}$, then wedging the right-hand side of (6) with $g e_{p_{0}}$ one obtains $\pi_{n^{\prime}, p_{0}}(g \omega)$, which projects to $\pi_{n_{0}, p_{0}}(g \omega) \notin \mathrm{X}_{n_{0}, p_{0}}$. This element is also obtained from the lefthand side by applying $\pi_{n_{0}, p_{0}-1}$ and then wedging with the projection of $g e_{p_{0}}$ to $V_{n_{0}, p_{0}}^{*}$. Hence the element $\pi_{n_{0}, p_{0}-1} g^{\prime} \omega$ does not lie in $\mathrm{X}_{n_{0}, p_{0}-1}$.

Corollary 7.2. Let $\mathbf{X}$ be a bounded Plücker variety. Then there exist $n_{0}, p_{0} \in \mathbb{Z}_{\geq 0}$ such that for all $n, p \in \mathbb{Z}_{\geq 0}$, and all $\omega \in \bigwedge^{p} V_{n, p}^{*}$, the following are equivalent:

(1) $\omega \notin X_{n, p}$.

(2) There exists $g \in G_{n, p}$ such that $\pi_{\min \left(n, n_{0}\right), \min \left(p, p_{0}\right)}(g \omega) \notin X_{\min \left(n, n_{0}\right), \min \left(p, p_{0}\right)}$.

Proof. By Corollary 6.6, there exist $n_{0}, p_{0}$ such that for all $n, p$ and $\omega \in \bigwedge^{p} V_{n, p}^{*}$ we have $\omega \notin X_{n, p}$ if and only if $\exists g \in G_{\infty}: \pi_{n_{0}, p_{0}}(g \omega) \notin X_{n_{0}, p_{0}}$. Now apply Lemma 7.1 repeatedly to get $g$ down to $G_{n, p}$.

We conclude this section with the proof of Theorem 1.3 .

Proof of Theorem 1.3. Let $n_{0}, p_{0}$ be as in the previous corollary, and let $f_{1}, \ldots, f_{N}$ be defining equations for $\mathrm{X}_{n_{0}, p_{0}}$.

Let $\left(d, p, \omega \in \bigwedge^{p} K^{d}\right)$ be the input to the algorithm. We first give a randomised algorithm for testing wether $\omega \in \mathbf{X}_{p}\left(K^{d}\right)$. First, if $p>d$, then $\omega=0$ and the output is yes if $\mathbf{X}$ is not the empty Plücker variety and no if it is. Otherwise, set $n:=d-p$, pick a random linear isomorphism $g: K^{d} \rightarrow V_{n, p}^{*}$, and return the answer to the question whether $f_{k}\left(\pi_{n_{0}, p_{0}} \bigwedge^{p} g(\omega)\right)=0$ for all $k=1, \ldots, N$.

If $\omega$ lies in $\mathbf{X}_{p}\left(K^{d}\right)$, then the output will always be yes. If $\omega$ does not lie in $\mathbf{X}_{p}\left(K^{d}\right)$, then by Corollary 7.2 an open and dense set of choices for $g$ will yield the correct output no. Clearly, the number of arithmetic operations over $K$ is polynomially bounded. Moreover, since the $f_{k}$ are fixed polynomials, no superpolynomial coefficient blow-up can happen if one works over $\mathbb{Q}$ or a more general number field. 
To make this algorithm deterministic, one can take the matrix entries $g_{i j}$ of $g$ to be variables rather than elements of $K$, and output yes if all $f_{k}\left(\pi_{n_{0}, p_{0}} \Lambda^{p} g(\omega)\right)$ are zero as polynomials in those variables. Now the arithmetic operations take place in the polynomial ring $K\left[g_{i j}\right]$, but (again since the $f_{i}$ are fixed) they still reduce to polynomially many operations over $K$, and to an algorithm of polynomial bitcomplexity over $\mathbb{Q}$ or number fields.

\section{Noetherianity of Matrix tUples}

We recall the statement of Theorem 1.5 . for all $p, n, m, d \in \mathbb{Z}_{\geq 0}$, the space

$$
A_{p, n, m, d}=\left(\operatorname{Mat}_{\mathbb{N}, \mathbb{N}}\right)^{p} \times \operatorname{Mat}_{\mathbb{N}, n} \times \operatorname{Mat}_{m, \mathbb{N}} \times K^{d}
$$

is $\mathrm{GL}_{\mathbb{N}} \times \mathrm{GL}_{\mathbb{N}}$-Noetherian with respect to the Zariski topology.

In the case where $p=0$, a much stronger statement is known to hold: the coordinate ring of this variety is $\operatorname{Sym}(\mathbb{N}) \times \operatorname{Sym}(\mathbb{N})$-Noetherian $\underline{\mathrm{HS} 12}$. But this fails for $p>0$ [HS12, Example 3.8], and we will not need this result in our proof. The entire section will be devoted to the proof of the theorem. We order $\mathbb{Z}_{\geq 0}^{4}$ lexicographically, and we apply induction on $(p, n, m, d)$. From here on, we assume that $A_{p^{\prime}, n^{\prime}, m^{\prime}, d^{\prime}}$ is $\mathrm{GL}_{\mathbb{N}} \times \mathrm{GL}_{\mathbb{N}^{-}}$Noetherian whenever $\left(p^{\prime}, n^{\prime}, m^{\prime}, d^{\prime}\right)$ is lexicographically smaller than $(p, n, m, d)$. We abbreviate $A=A_{p, n, m, d}$. For $x \in A$ we write $x_{\mathrm{ma}}, x_{\mathrm{col}}$, and $x_{\text {row }}$ for the projections of $x$ in $\left(\operatorname{Mat}_{\mathbb{N}, \mathbb{N}}\right)^{p}, \operatorname{Mat}_{\mathbb{N}, n}, \operatorname{Mat}_{m, \mathbb{N}}$, respectively.

A key step in our proof will be a version of the the following dichotomy: a $\mathrm{GL}_{\mathbb{N}} \times \mathrm{GL}_{\mathbb{N}}$-stable closed subset of $\left(\mathrm{Mat}_{\mathbb{N}, \mathbb{N}}\right)^{p}$ is either equal to $\left(\mathrm{Mat}_{\mathbb{N}, \mathbb{N}}\right)^{p}$ or else consists of matrix tuples of bounded rank, in the sense of the following definition.

Definition 8.1. For a tuple $M=\left(M_{1}, \ldots, M_{p}\right)$ of matrices of the same size (infinite or not), we define the rank as

$$
\operatorname{rk}(M)=\min \left\{\operatorname{rk}\left(c_{1} M_{1}+\ldots+c_{p} M_{p}\right) \mid\left(c_{1}: \cdots: c_{p}\right) \in \mathbb{P}^{p-1}\right\} \in \mathbb{Z}_{\geq 0} \cup\{\infty\} .
$$

Before establishing the dichotomy, we settle the bounded-rank case by induction.

Lemma 8.2. Fix $r \in \mathbb{Z}_{\geq 0}$. Then $\left\{x \in A \mid \operatorname{rk} x_{\mathrm{ma}} \leq r\right\}$ is $\mathrm{GL}_{\mathbb{N}} \times \mathrm{GL}_{\mathbb{N}}$-Noetherian.

Proof. Consider the morphism $\varphi: A_{p-1, n+r, m+r, d+p^{2}} \rightarrow A_{p, n, m, d}=A$ defined as follows. Let $\left(M_{1}, \ldots, M_{p-1}\right) \in \mathrm{Mat}_{\mathbb{N}, \mathbb{N}}^{p-1}$, let $C_{1} \in \operatorname{Mat}_{\mathbb{N}, m}, C_{2} \in \operatorname{Mat}_{\mathbb{N}, r}$, let $R_{1} \in \operatorname{Mat}_{m, \mathbb{N}}, R_{2} \in \operatorname{Mat}_{r, \mathbb{N}}$, and let $t \in K^{d}$ and $\left(\alpha_{i j}\right) \in K^{p \times p}$. Then

$$
\varphi\left(\left(M_{1}, \ldots, M_{p-1}\right),\left(C_{1}, C_{2}\right),\left(R_{1}, R_{2}\right),(t, \alpha)\right):=\left(x_{\mathrm{ma}}, C_{1}, R_{1}, t\right)
$$

where the $i$-th matrix in $x_{\text {ma }}$ equals

$$
\sum_{j=1}^{p-1} \alpha_{i j} M_{j}+\alpha_{i p} C_{2} \cdot R_{2}
$$

This map is $\mathrm{GL}_{\mathbb{N}} \times \mathrm{GL}_{\mathbb{N}}$-equivariant, continuous, and its image equals the set in the statement of the lemma. Since $\left(p-1, n+r, m+r, d+p^{2}\right)$ is lexicographically smaller than $(p, n, m, d)$, the left-hand space is equivariantly Noetherian by the induction assumption. Hence so is its image.

Similar induction arguments apply to the set of $x \in A$ for which $x_{\mathrm{col}} \in$ Mat $_{\mathbb{N}, n}$ has rank strictly less than $n$ or $x_{\text {row }}$ has rank strictly less than $m$. So we need only focus on the $x$ for which $x_{\text {col }}$ and $x_{\text {row }}$ have full rank, and $x_{\text {ma }}$ has high rank. We start with an easy lemma in linear algebra. 
Lemma 8.3. Let $N_{1}, N_{2} \in \mathbb{Z}_{\geq 0}$, and let $M \in\left(\operatorname{Mat}_{N_{1}, N_{2}}\right)^{p}$. If $M$ has rank at least $p$, then there exists a $v \in K^{N_{2}}$ for which $M_{1} v, \ldots, M_{p} v \in K^{N_{1}}$ are linearly independent.

Proof. Consider the variety

$$
Y:=\left\{(v, d) \in K^{N_{2}} \times \mathbb{P}^{p-1} \mid\left(d_{1} M_{1}+\ldots+d_{p} M_{p}\right) \cdot v=0\right\} .
$$

Given $d \in \mathbb{P}^{p-1}$, the space $\left\{v \mid\left(v,\left(d_{i}\right)_{i=1}^{p}\right) \in Y\right\}$ has dimension at most $N_{2}-p$ because $\operatorname{rk}\left(d_{1} M_{1}+\ldots+d_{p} M_{p}\right) \geq p$ by assumption. In other words, the fibre in $Y$ above $d$ has dimension at most $N_{2}-p$. But then $Y$ has dimension at most $N_{2}-p+p-1<N_{2}$, and hence the projection from $Y$ to $K^{N_{2}}$ is not surjective.

Corollary 8.4. Let $N_{1}, N_{2} \in \mathbb{Z}_{\geq 0}$, let $M \in\left(\operatorname{Mat}_{N_{1}, N_{2}}\right)^{p}$, and let $l \in \mathbb{Z}_{\geq 0}$. If $M$ has rank at least $p l$, then there exists a linear subspace $V \subseteq K^{N_{2}}$ of dimension $l$ such that $M_{1} V+\ldots+M_{p} V$ has dimension $p l$.

Proof. We apply induction. For $l=1$ the corollary follows from Lemma 8.3. Let $l>1$, and assume that the corollary is true for $l-1$. Use the lemma to pick $v \in K^{N_{2}}$ such that $W:=\left\langle M_{1} v, \ldots, M_{p} v\right\rangle$ has dimension $p$. Then each $M_{i}$ induces a linear map

$$
\tilde{M}_{i}: K^{N_{2}} /\langle v\rangle \rightarrow K^{N_{1}} / W
$$

and the tuple $\left(\tilde{M}_{1}, \ldots, \tilde{M}_{p}\right)$ has rank at least $p l-p=p(l-1)$-indeed, deleting a row or column from a matrix tuple reduces the rank by at most one, and deleting a zero row from a matrix tuple does not reduce the rank. By the induction hypothesis we find an $(l-1)$-dimensional $V^{\prime} \subseteq K^{N_{2}} /\langle v\rangle$ such that $\operatorname{dim}\left(\tilde{M}_{1} V^{\prime}+\cdots+\tilde{M}_{p} V^{\prime}\right)=$ $p(l-1)$, and the preimage $V$ of $V^{\prime}$ in $K^{N_{2}}$ has the desired property.

Corollary 8.5. Let $M \in\left(\operatorname{Mat}_{\mathbb{N}, \mathbb{N}}\right)^{p}$ and let $l \in \mathbb{Z}_{\geq 0}$. If $M$ has rank at least pl, then there exists a linear space $V \subseteq K^{(\mathbb{N})}$ of dimension l such that $M_{1} V+\ldots+M_{p} V$ has dimension $p l$.

Here, by $K^{(\mathbb{N})}$ we mean the countable-dimensional subspace of $K^{\mathbb{N}}$ where all but finitely many coordinates are zero. A matrix in Mat $_{\mathbb{N}, \mathbb{N}}$ defines naturally a linear map $K^{(\mathbb{N})} \rightarrow K^{\mathbb{N}}$, which is referred to in the corollary.

Proof. For $N \in \mathbb{Z}_{\geq 0}$, denote by $\pi_{N}$ the projection from Mat $_{\mathbb{N}, \mathbb{N}}$ to $\operatorname{Mat}_{N, N}$. Define the variety

$$
D_{N}=\left\{\left(d_{1}: \ldots: d_{p}\right) \in \mathbb{P}^{p-1} \mid \operatorname{rk}\left(\pi_{N}\left(d_{1} M_{1}+\ldots+d_{p} M_{p}\right)\right)<p l\right\} .
$$

Observe that $D_{1} \supseteq D_{2} \supseteq \ldots$ is a descending sequence of closed subvarieties of $\mathbb{P}^{p-1}$. Moreover, the intersection of the $D_{N}$ is $\emptyset$ because $M$ has rank at least $p l$. So there exists an $N \in \mathbb{Z}_{\geq 0}$ such that $\operatorname{rk}\left(\pi_{N}\left(M_{1}\right), \ldots, \pi_{N}\left(M_{p}\right)\right) \geq p l$. Now apply Corollary 8.4 to find a linear subspace $V \subseteq K^{N}$ such that $\pi_{N}\left(M_{1}\right) V+\ldots+\pi_{N}\left(M_{p}\right) V$ has dimension $p l$. View $V$ as a subspace of $K^{(\mathbb{N})}$, and observe that $M_{1} V+\ldots+M_{p} V$ has dimension $p l$, as desired.

For $N_{1}, N_{2} \in \mathbb{Z}_{\geq 0}$, we denote $A_{\text {fin }}^{N_{1}, N_{2}}:=\left(\operatorname{Mat}_{N_{1}, N_{2}}\right)^{p} \times \operatorname{Mat}_{N_{1}, n} \times \operatorname{Mat}_{m, N_{2}}$. Note that we have a natural projection from $A$ to $A_{\text {fin }}^{N_{1}, N_{2}}$.

Proposition 8.6. Let $N_{1}, N_{2} \in \mathbb{Z}_{\geq 0}$. Then there exists an $r \in \mathbb{Z}_{\geq 0}$ such that for any $x \in A$ with $\operatorname{rk}\left(x_{\mathrm{ma}}\right) \geq r$ and with $x_{\mathrm{col}}$ and $x_{\mathrm{row}}$ of full rank, the projection of the orbit $\mathrm{GL}_{\mathbb{N}} \times \mathrm{GL}_{\mathbb{N}} x$ to $A_{\mathrm{fin}}^{N_{1}, N_{2}}$ is dense in $A_{\mathrm{fin}}^{N_{1}, N_{2}}$. 
Proof. It suffices to prove the lemma for $N_{1}=p l$ and $N_{2}=l$ with $l \in \mathbb{Z}_{\geq 0}$. Let $r:=n+m+p l$ and let $x \in A$ be as in the statement of the proposition. As in Corollary 8.5. there exists an $N$ such that the projection of $x_{\text {ma }}$ to the first $N \times N$ coordinates has rank at least $r$. Without loss of generality (taking $N$ larger if necessary), we assume that the projection of $x_{\mathrm{col}}$ in Mat $_{N, n}$ and the projection of $x_{\text {row }}$ in $\mathrm{Mat}_{m, N}$ have full rank. From now on, we view $x$ as an element of $A_{\text {fin }}^{N, N}$, and only act on it with elements of $\mathrm{GL}_{N} \times \mathrm{GL}_{N}$ - the first copy on $x_{\mathrm{ma}}, x_{\mathrm{col}}$ by row operations, and the second copy on $x_{\text {ma }}, x_{\text {row }}$ by column operations.

Without loss of generality, we may assume that

$$
x_{\mathrm{col}}=\left[\begin{array}{c}
0_{N-n, n} \\
I_{n}
\end{array}\right] \text { and } x_{\mathrm{row}}=\left[\begin{array}{ll}
0_{m, N-m} & I_{m}
\end{array}\right] .
$$

Let $M^{\prime}=x_{\mathrm{ma}}^{\prime}$ be the projection of $M=x_{\mathrm{ma}}$ to $\left(\operatorname{Mat}_{N-n, N-m}\right)^{p}$. Observe that $M^{\prime}$ has rank at least $p l$. Then by Corollary 8.4 there exists a linear subspace $V \subset K^{N-m}$ of dimension $l$ such that $W:=M_{1}^{\prime} V+M_{2}^{\prime} V+\ldots+M_{p}^{\prime} V \subset K^{N-n}$ has dimension $p l$. View $V$ as a subspace of $K^{N}$. Performing column operations on the first $N-m$ columns does not change $x_{\text {row }}$ and can bring $V$ into the span of the first $l$ standard basis elements. Performing row operations on the first $N-m$ rows does not change $x_{\mathrm{col}}$ and can bring $W$ into the span of the first $p l$ standard basis vectors. Performing further row operations on the first $p l$ rows we achieve that

$$
x_{\mathrm{ma}}=\left(\left[\begin{array}{cc}
I_{l} & *_{l, N-l} \\
0_{l} & *_{l, N-l} \\
\vdots & \vdots \\
0_{l} & *_{l, N-l} \\
*_{N-p l, l} & *_{N-p l, N-l}
\end{array}\right],\left[\begin{array}{cc}
0_{l} & * \\
I_{l} & * \\
\vdots & \vdots \\
0_{l} & * \\
* & *
\end{array}\right], \ldots,\left[\begin{array}{cc}
0_{l} & * \\
0_{l} & * \\
\vdots & \vdots \\
I_{l} & * \\
* & *
\end{array}\right]\right) .
$$

Subtracting suitable linear combinations of the first $p l$ rows from the last $N-p l$ rows, we can clear the $*$ s below the identity matrices:

$$
x_{\mathrm{ma}}=\left(\left[\begin{array}{cc}
I_{l} & *_{l, N-l} \\
0_{l} & *_{l, N-l} \\
\vdots & \vdots \\
0_{l} & *_{l, N-l} \\
0_{N-p l, l} & *_{N-p l, N-l}
\end{array}\right],\left[\begin{array}{cc}
0_{l} & * \\
I_{l} & * \\
\vdots & \vdots \\
0_{l} & * \\
0 & *
\end{array}\right], \ldots,\left[\begin{array}{cc}
0_{l} & * \\
0_{l} & * \\
\vdots & \vdots \\
I_{l} & * \\
0 & *
\end{array}\right]\right),
$$

still with $x_{\text {row }}$ and $x_{\text {col }}$ as above. To see that the $\mathrm{GL}_{N} \times \mathrm{GL}_{N}$-orbit of $x$ projects dominantly into $A_{\text {fin }}^{p l, l}$, pick a general point $x^{\prime}$ in the latter space. Subtract a linear combination of the last $N-m$ columns of $x_{\text {ma }}$ and $x_{\text {row }}$ from the first $l$ columns to achieve that $x_{\text {row }}$ becomes equal to $x_{\text {row }}^{\prime}$. This messes up the $0 / I$-structure of $x_{\text {ma }}$, but by generality of $x_{\text {col }}^{\prime}$ we may assume that the $p l \times p l$-matrix obtained from the new $x_{\mathrm{ma}}$ by concatenating the $p l \times l$-submatrices of the $p$ components is still invertible (though no longer the identity matrix). Moreover, we may assume that the same holds for $x_{\mathrm{ma}}^{\prime}$. Then suitable row operations with the first $p l$ rows move $x_{\mathrm{ma}}$ into $x_{\mathrm{ma}}^{\prime}$, and subtracting suitable multiples of these $p l$ rows from the $N-p l$ rows below them again clears the $*^{\prime} s$. Finally, subtracting a suitable linear combination of the last $N-n$ rows from the first $p l$ rows fixes $x_{\mathrm{ma}}=x_{\mathrm{ma}}^{\prime}$ and $x_{\mathrm{col}}=x_{\mathrm{col}}^{\prime}$ but moves $x_{\text {row }}$ into $x_{\text {row }}^{\prime}$.

Here is the promised dichotomy. 
Corollary 8.7. Let $Y$ be $a \mathrm{GL}_{\mathbb{N}} \times \mathrm{GL}_{\mathbb{N}}$-stable closed subset of $\left(\mathrm{Mat}_{\mathbb{N}, \mathbb{N}}\right)^{p} \times \mathrm{Mat}_{\mathbb{N}, n} \times$ Mat $_{m, \mathbb{N}}$. If $Y$ contains elements $x$ with $x_{\text {row }}$ and $x_{\text {col }}$ of full rank and $x_{\text {ma }}$ of arbitrarily high rank, then $Y=\left(\operatorname{Mat}_{\mathbb{N}, \mathbb{N}}\right)^{p} \times \operatorname{Mat}_{\mathbb{N}, n} \times \operatorname{Mat}_{m, \mathbb{N}}$.

Proof. Let $f$ be a polynomial that vanishes identically on $Y$. Then there exist $N_{1}, N_{2}$ such that the matrix entries appearing in $f$ are coordinates on $A_{\text {fin }}^{N_{1}, N_{2}}$. Let $r$ be as in the proposition, and pick an element $x \in Y$ with $\operatorname{rk} x_{\mathrm{ma}}=r$ and $x_{\mathrm{col}}, x_{\mathrm{row}}$ of full rank. Then $f$ vanishes on the projection of $\mathrm{GL}_{\mathbb{N}} \times \mathrm{GL}_{\mathbb{N}} x$ in $A_{\mathrm{fin}}^{N_{1}, N_{2}}$. Hence, by the proposition, $f$ is zero.

Now we can complete the proof of the theorem.

Proof of Theorem 1.5. Assume that we have a descending chain

$$
A \supseteq Y_{1} \supseteq Y_{2} \supseteq \ldots
$$

of $\mathrm{GL}_{\mathbb{N}} \times \mathrm{GL}_{\mathbb{N}}$-stable closed subsets of $A$. For $r \in \mathbb{Z}_{\geq 0}$ let $U_{k}^{r}$ denote the subset of $Y_{k}$ where $x_{\text {col }}$ and $x_{\text {row }}$ have full rank and $x_{\text {ma }}$ has rank at least $r$. By Lemma 8.2 (and variations concerning $x_{\mathrm{col}}$ and $x_{\text {row }}$ ), it suffices to prove that for some value of $r$, the chain of closures

$$
A \supseteq \overline{U_{1}^{r}} \supseteq \overline{U_{2}^{r}} \supseteq \ldots
$$

stabilises. Note that $U_{k}^{r}$ shrinks both when $k$ grows and when $r$ grows. Since $K^{d}$ is Noetherian, we may choose $k$ and $r$ such that for all $l \geq k$ and $s \geq r$ the closure of the projection of $U_{l}^{s}$ into $K^{d}$ is constant, say equal to $Z$. We will then argue that

$$
\overline{U_{l}^{r}}=\left(\operatorname{Mat}_{\mathbb{N}, \mathbb{N}}\right)^{p} \times \operatorname{Mat}_{\mathbb{N}, n} \times \operatorname{Mat}_{m, \mathbb{N}} \times Z \text { for all } l \geq k .
$$

To simplify notation, write $X:=U_{l}^{r}$, and let $f$ be a polynomial function on $A$ that vanishes identically on $X$. We can write $f=\sum_{i=1}^{l} f_{i} \otimes h_{i}$ with each $f_{i}$ defined on $\left(\operatorname{Mat}_{\mathbb{N}, \mathbb{N}}\right)^{p} \times \operatorname{Mat}_{\mathbb{N}, n} \times$ Mat $_{m, \mathbb{N}}$, and each $h_{i}$ defined on $K^{d}$, and with the $f_{i}$ linearly independent. We will argue that each $h_{i}$ vanishes on $Z$.

Choose $N_{1}, N_{2}$ such that all $f_{i}$ involve only coordinates from $A_{\text {fin }}^{N_{1}, N_{2}}$. By the proposition there exists an $s \geq r$ such that for all $x \in\left(\operatorname{Mat}_{\mathbb{N}, \mathbb{N}}\right)^{p} \times \operatorname{Mat}_{\mathbb{N}, n} \times$ Mat $_{m, \mathbb{N}}$ with $x_{\text {col }}, x_{\text {row }}$ of full rank and $\operatorname{rk} x_{\text {ma }} \geq s$ the orbit $\mathrm{GL}_{\mathbb{N}} \times \mathrm{GL}_{\mathbb{N}} x$ projects dominantly into $A_{\text {fin }}^{N_{1}, N_{2}}$. In particular, the restrictions of the $f_{i}$ to $\mathrm{GL}_{\mathbb{N}} \times \mathrm{GL}_{\mathbb{N}} x$ are still $K$-linearly independent. Now if such an $x$ lies in the fibre in $X$ over $z \in Z$, then we find

$$
0=f(g(x, z))=f(g x, z)=\sum_{i} f_{i}(g x) h_{i}(z) \text { for all } g \in G_{\infty}
$$

and by varying the $g$ we conclude that $h_{i}(z)=0$ for all $i$. Finally, the image of $U_{l}^{s} \subseteq X$ in $Z$ is dense by assumption, and hence $h_{i}(z)=0$ for all $i$ and $z$.

\section{Discussion}

We have introduced the natural notion of Plücker variety, which is a family of subvarieties of exterior powers that, like Grassmannians, are functorial and behave well under duals. For the bounded ones among these, we have established that they are defined by polynomial equations of bounded degree, independently of the particular instance of the Plücker variety. This result is new already for the first secant variety of the Grassmannian, and for the tangential variety of the Grassmannian. 
Before turning to several open questions that result from our work, let us explain the second part of the title of this paper. In Section 4 we have seen that the projective limit of all (cones over) Grassmannians is (the cone over) Sato's Grassmannian. Higher secant varieties of any Plücker variety are again Plücker varieties, of which one can take the limit. But, in fact, passing to the limit commutes with taking joins, and in particular with taking secant varieties. In other words, for Plücker varieties $\mathbf{X}$ and $\mathbf{Y}$ the limit of $\mathbf{X}+\mathbf{Y}$ as defined in Section 4 equals the closure of the set of all points of the form $x+y$ with $x \in \mathrm{X}_{\infty}$ and $y \in Y_{\infty}$, where the addition takes place in the dual infinite wedge vector space $\left(\bigwedge^{\frac{\infty}{2}} V_{\infty}\right)^{*}$. To see this one uses the right-hand side of Diagram 5, which lifts all instances of $X$ to a dense subset of $\mathrm{X}_{\infty}$. Thus a special case of our main theorem is that higher secant varieties of Sato's Grassmannian are defined set-theoretically by finitely many $G_{\infty}$-orbits of equations. Another result that one easily derives from the Noetherianity of matrix tuples is that, for any number $p$, the Cartesian product of $p$ copies of Sato's Grassmannian, with the Zariski-topology, is equivariantly Noetherian with respect to a single copy of $G_{\infty}$ acting diagonally. We conclude with a number of open problems.

(1) Is there an ideal-theoretic analogue of our main theorem? This is a very interesting, but apparently also very difficult question. It is not true that the ideal of the limit of every bounded Plücker variety is generated by finitely many $G_{\infty}$-orbits of equations. Indeed, using the fact that the ideal of the finite-dimensional Grassmannian in $\bigwedge^{p} V$ is generated by a number of $\mathrm{GL}(V)$-modules in the symmetric power $S^{2} \bigwedge^{p} V^{*}$ that is unbounded as $p$ and $\operatorname{dim} V-p$ grow, one can show that the ideal of Sato's Grassmannian is not generated by any finite number of $G_{\infty}$-orbits of polynomials. Thus any progress on the ideal-theoretic question would require entirely new ideas. This is different from the situation for ordinary tensors, where at least a conjectural ideal-theoretic analogue of our theorems exists DK14]. A closely related question is how to generalise Snowden's $\Delta$-modules Sno13. to modules of syzygies for Plücker varieties. An intermediate question, between our set-theoretic result and an ideal-theoretic analogue, is whether a variant of our main theorem holds in the setting of projective schemes; we do not know the answer to this question either.

(2) Most Plücker varieties of interest to us are constructed from Grassmannians by operations such as joins and tangential varieties. These are all bounded. Nevertheless, the restriction to bounded Plücker varieties in Theorems 1.1 and 1.3 seems somewhat ad hoc. Are these theorems true for unbounded Plücker varieties, as well? Our proof in Section 6 uses the recursive nature of Pfaffians in Section 5 in a fundamental manner. It is conceivable that, for general Plücker varieties, this structure can be replaced with techniques like prolongation that produce equations for higher secant varieties given equations for lower secant varieties [SS09, CJ96]. A second important ingredient in the proofs is the Noetherianity of matrix tuples, Theorem 1.5 . We may need an analogue of this for higher-dimensional tensors to deal with general Plücker varieties.

(3) Is the ideal-theoretic version of Theorem 1.5 true? This question seems easier than the preceding questions, and we conjecture that the answer is positive. This question is essentially a question about two-variable tca's in the sense of [SS12a, SS12b]. 


\section{REFERENCES}

[Abe80] Silvana Abeasis. On the Plücker relations for the Grassmann varieties. Advances in Mathematics, 36(3):277 - 282, 1980.

[BB14] Weronika Buczyńska and Jarosław Buczyński. Secant varieties to high degree Veronese reembeddings, catalecticant matrices and smoothable Gorenstein schemes. J. Algebr. Geom., 23(1):63-90, 2014.

[BC03] Winfried Bruns and Aldo Conca. Gröbner bases and determinantal ideals. In Commutative algebra, singularities and computer algebra. Proceedings of the NATO advanced research workshop, Sinaia, Romania, September 17-22, 2002, pages 9-66. Dordrecht: Kluwer Academic Publishers, 2003.

[BDdG07] Karin Baur, Jan Draisma, and Willem A. de Graaf. Secant dimensions of minimal orbits: computations and conjectures. Exp. Math., 16(2):239-250, 2007.

[BO00] Spencer Bloch and Andrei Okounkov. The character of the infinite wedge representation. Adv. Math., 149(1):1-60, 2000.

$\left[\mathrm{CDD}^{+} 14\right]$ A. Conca, S. Di Rocco, J. Draisma, J. Huh, B. Sturmfels, and F. Viviani. Combinatorial algebraic geometry. Lecture notes of the CIME-CIRM summer school, Levico Terme, Italy, June 10-15, 2013. Berlin: Springer, 2014.

[CEF15] Thomas Church, Jordan S. Ellenberg, and Benson Farb. FI-modules: a new approach to stability for $S_{n}$-representations. Duke Math. J., 2015. To appear, arxiv.org: 1204.4533

[CGG05] Maria V. Catalisano, Anthony V. Geramita, and Alessandro Gimigliano. Secant varieties of Grassmann varieties. Proc. Am. Math. Soc., 133(3):633-642, 2005.

[CJ96] Michael L. Catalano-Johnson. The possible dimensions of the higher secant varieties. Am. J. Math., 118(2):355-361, 1996.

[DE15] Jan Draisma and Rob H. Eggermont. Finiteness results for Abelian tree models. J. Eur. Math. Soc., 17:711-738, 2015.

[DEKL13] Jan Draisma, Rob H. Eggermont, Robert Krone, and Anton Leykin. Noetherianity for infinite-dimensional toric varieties. June 2013. Available from http://arxiv.org/abs/1306.0828.

[DK14] Jan Draisma and Jochen Kuttler. Bounded-rank tensors are defined in bounded degree. Duke Math. J., 163(1):35-63, 2014.

[Dra10] Jan Draisma. Finiteness for the k-factor model and chirality varieties. Adv. Math., 223:243-256, 2010.

[HMSV09] Benjamin Howard, John Millson, Andrew Snowden, and Ravi Vakil. The equations for the moduli space of $n$ points on the line. Duke Math. J., 146(2):175-226, 2009.

[HS12] Christopher J. Hillar and Seth Sullivant. Finite Gröbner bases in infinite dimensional polynomial rings and applications. Adv. Math., 229(1):1-25, 2012.

[KPRS08] Alex Kasman, Kathryn Pedings, Amy Reiszl, and Takahiro Shiota. Universality of rank 6 Plücker relations and Grassmann cone preserving maps. Proc. Amer. Math. Soc., 136:77-87, 2008.

[LM04] Joseph M. Landsberg and Laurent Manivel. On the ideals of secant varieties of Segre varieties. Found. Comput. Math., 4(4):397-422, 2004.

[LO13] Joseph M. Landsberg and Giorgio Ottaviani. Equations for secant varieties of Veronese and other varieties. Ann. Mat. Pura Appl. (4), 192(4):569-606, 2013.

[Mac01] Diane MacLagan. Antichains of monomial ideals are finite. Proc. Am. Math. Soc., 129(6):1609-1615, 2001.

[MM14] L. Manivel and M. Michałek. Secants of minuscule and cominuscule minimal orbits. January 2014. Available from http://arxiv.org/abs/1401.1956.

[Qi13] Yang Qi. Equations for the third secant variety of the Segre product of $n$ projective spaces. 2013. Available from http://arxiv.org/abs/1311.2566.

[Rai12] Claudiu Raicu. Secant varieties of Segre-Veronese varieties. Algebra Number Theory, 6(8):1817-1868, 2012.

[RZ13] Rodolfo Rios-Zertuche. An introduction to the half-infinite wedge. 2013. Available from http://arxiv.org/abs/1309.0054.

[Sno13] Andrew Snowden. Syzygies of Segre embeddings and $\Delta$-modules. Duke Math. J., 162(2):225-277, 2013. 
[SS83] Mikio Sato and Yasuko Sato. Soliton equations as dynamical systems on infinite dimensional Grassmann manifold. Nonlinear partial differential equations in applied science, Proc. U.S. - Jap. Semin., Tokyo 1982, North-Holland Math. Stud. 81, 259-271 (1983)., 1983.

[SS09] Jessica Sidman and Seth Sullivant. Prolongations and computational algebra. Can. J. Math., 61(4):930-949, 2009.

[SS12a] Steven V. Sam and Andrew Snowden. GL-equivariant modules over polynomial rings in infinitely many variables. June 2012. Available from http://arxiv.org/abs/1206.2233.

[SS12b] Steven V. Sam and Andrew Snowden. Introduction to twisted commutative algebras. September 2012. Available from http://arxiv.org/abs/1402.1667.

[Str83] Volker Strassen. Rank and optimal computation of generic tensors. Linear Algebra Appl., 52-53:645-685, 1983.

[SW85] Graeme Segal and George Wilson. Loop groups and equations of KdV type. Publ. Math., Inst. Hautes Étud. Sci., 61:5-65, 1985.

[VMP98] A. Álvarez Vázquez, J.M. Muñoz Porras, and F.J. Plaza Martín. The algebraic formalism of soliton equations over arbitrary base fields. In Taller de variedades abelianas y funciones theta. Morelia, México, 1996. Memorias, pages 3-40. México: Sociedad Matemática Mexicana, 1998.

(Jan Draisma) Department of Mathematics and Computer Science, Technische Universiteit Eindhoven, P.O. Box 513, 5600 MB Eindhoven, The Netherlands; and Vrije Universiteit and Centrum voor Wiskunde en Informatica, Amsterdam, The Netherlands E-mail address: j.draisma@tue.nl

(Rob H. Eggermont) Department of Mathematics and Computer Science, Technische Universiteit Eindhoven, P.O. Box 513, 5600 MB Eindhoven, The Netherlands

E-mail address: r.h.eggermont@tue.nl 